\title{
Genome-scale functional profiling of cell cycle controls in African trypanosomes
}

2

3

4

${ }^{1}$ Wellcome Trust Centre for Anti-Infectives Research, School of Life Sciences, University of Dundee, United Kingdom

2 Tayside Centre for Genomic Analysis, Ninewells Hospital and School of Medicine, University of Dundee, United Kingdom

${ }^{3}$ Current address: Wellcome Trust Centre for Integrative Parasitology, University of Glasgow, United Kingdom

$\S$ joint first authors

Keywords: checkpoint, kinetoplastid, RIT-seq, sleeping sickness, Leishmania, Trypanosoma brucei, Trypanosoma cruzi. 


\section{Abstract}

33 Trypanosomatids, which include major pathogens of humans and livestock, are divergent 34 eukaryotes for which cell cycle controls and the underlying mechanisms are not completely understood. Here, we describe a genome-wide RNA-interference library screen for cell cycle regulators in bloodstream form Trypanosoma brucei. We induced massive parallel knockdown and sorted the perturbed population into cell cycle stages using flow cytometry. RNAi-targets were deep-sequenced from each stage and cell cycle profiles were digitally reconstructed at a genomic scale. We identify hundreds of proteins that impact cell cycle progression; glycolytic enzymes required for $\mathrm{G}_{1} \mathrm{~S}$ progression, DNA replication factors, mitosis regulators, proteasome and kinetochore complex components required for $\mathrm{G}_{2} \mathrm{M}$ progression, flagellar and cytoskeletal components required for cytokinesis, mRNA-binding factors, protein kinases and many previously uncharacterised proteins. The outputs facilitate functional annotation and drug-target prioritisation and provide comprehensive functional genomic evidence for the machineries, pathways and regulators that coordinate progression

46 through the trypanosome cell cycle. visualization tool (https://tryp-cycle.onrender.com). 


\section{Introduction}

The canonical eukaryotic cell cycle encompasses discrete phases: $\mathrm{G}_{1}$ (gap 1), when the cell prepares for DNA replication; S (synthesis) phase, when nuclear DNA replication takes place; $\mathrm{G}_{2}$ (gap 2), when the cell prepares for mitosis; and M (mitosis) when the replicated DNA is segregated and the nucleus divides (1). Mitosis is immediately followed by cytokinesis (cell division), generating two daughter cells (2). Anomalies occurring during cell cycle progression can result in cell cycle arrest, to allow the cell to resolve the anomaly; in cell death, if the anomaly cannot be resolved or, among other outcomes, carcinogenesis. Therefore, progression through the cell cycle is typically under strict checkpoint control; the $\mathrm{G}_{1}-\mathrm{S}$, intra $\mathrm{S}$ phase, $\mathrm{G}_{2}-\mathrm{M}$ and spindle checkpoints control the onset of $\mathrm{S}$ phase, $\mathrm{S}$ phase progression, the onset of $\mathrm{M}$ phase and $\mathrm{M}$ phase progression, respectively (3). These processes have been extensively studied, particularly because cell cycle defects are common triggers for carcinogenesis (4). However, our understanding of the evolution and mechanisms of eukaryotic cell cycle progression control derives primarily from studies on the opisthokonts (including animals and fungi), with relatively fewer studies on more divergent eukaryotes, such as the trypanosomatids (1).

The trypanosomatids are flagellated protozoa and include parasites that cause a range of neglected tropical diseases that have major impacts on human and animal health. The African trypanosome, Trypanosoma brucei, is transmitted by tsetse flies and causes both human and animal diseases, sleeping sickness and nagana, respectively, across subSaharan Africa (5). T. brucei has emerged as a highly tractable experimental system, both as a parasite and as a model organism (6). For example, the T. brucei flagellum (7) serves as a model for studies on human ciliopathies (8-11). Divergent features, shared with other pathogenic trypanosomatids, such as Trypanosoma cruzi and Leishmania, include glycolysis compartmentalised within glycosomes (12), a single mitochondrion with a complex mitochondrial DNA structure known as the kinetoplast (13) and polycistronic transcription of almost every gene (14). Widespread, and constitutive, polycistronic transcription in trypanosomatids places major emphasis on post-transcriptional controls by mRNA binding proteins (RBPs) and post-translational controls, involving protein phosphorylation in particular.

Studies that focus on cell cycle controls in $T$. brucei have revealed features that are conserved with other well-studied eukaryotes, but also features that are divergent $(13,15)$. Most notably, the available evidence suggest that certain cell cycle checkpoints are absent. For example, cytokinesis is not dependent upon either mitosis or nuclear DNA synthesis in the insect stage of $T$. brucei (16). Moreover, functions previously thought to be fulfilled by highly conserved proteins employ lineage-specific or highly divergent proteins in trypanosomatids. The kinetochore complex, which directs chromosome segregation, is 
88 trypanosomatid-specific, for example (17), while the origin recognition complex (ORC),

89 involved in DNA replication initiation, is highly divergent (18). In terms of high-throughput

90 studies, transcriptome (19) and proteome (20) monitoring during the $T$. brucei cell cycle

91 revealed hundreds of regulated mRNAs and proteins, while phosphoproteomic analysis

92 revealed dynamic phosphorylation of several RBPs (21). Nevertheless, divergence presents

93 a substantial challenge, many $T$. brucei genes have not yet been assigned a specific

94 function, and many cell cycle regulators likely remain to be identified.

High-throughput functional genetic screens can be used to simultaneously assess

96 every gene in a genome for a role in a particular process. We developed RNA interference

97 Target Sequencing (RIT-seq) for T. brucei (22) and previously generated genome-scale

98 fitness profiles, facilitating essentiality predictions and the prioritisation of potential drug

99 targets (23). Here we describe a genome-scale RIT-seq screen to identify cell cycle controls

100 and regulators in bloodstream form African trypanosomes. Following induction of

101 knockdown, the cells were sorted according to their DNA content using fluorescence-

102 activated cell sorting (FACS). The sorted populations were the $G_{1}, S$ and $G_{2} M$ cell cycle

103 stages as well as perturbed cell populations with either less DNA than typically found in $\mathrm{G}_{1}$

104 or more DNA than typically found in $\mathrm{G}_{2} \mathrm{M}$. RIT-seq analysis was then carried out for each

105 sorted population and cell cycle profiles were digitally reconstructed for each gene using

106 sequencing read-counts. This genome-wide screen reveals the protein complexes, pathways

107 and signalling factors that coordinate progressive steps through the trypanosome cell cycle,

108 both improving our understanding of trypanosome cell biology and also further facilitating the

109 prioritisation of new potential drug targets. 


\section{Results}

\section{A genome-wide conditional knockdown screen for cell cycle progression defects}

112 Bloodstream form T. brucei are readily grown in cell culture, with exponential

113 proliferation and a doubling time of approximately $6.5 \mathrm{~h}$. The $T$. brucei genome is diploid

114 such that $\mathrm{G}_{1}$ cells have a $2 \mathrm{C}$ genome content; $\mathrm{C}$ represents the haploid DNA content. Cells

115 progressing through nuclear $S$ phase, and replicating their DNA, have a genome content

116 between $2 \mathrm{C}$ and $4 \mathrm{C}$, while cells that have completed DNA replication $\left(\mathrm{G}_{2} \mathrm{M}\right)$ have a $4 \mathrm{C}$ DNA

117 content (Figure 1A). Cytokinesis then produces two daughter cells with a 2C DNA content.

118 Some perturbations can yield defects which become apparent when cytokinesis produces

119 sub-2C cells or over-replicated, polyploid (>4C) cells (Figure 1A). These polyploid cells arise

120 due to endoreduplication, additional rounds of DNA replication without cytokinesis, either

121 with $(24)$ or without $(25,26)$ mitosis, yielding cells with multiple diploid nuclei or cells with

122 polyploid nuclei, respectively.

123 We devised a high-throughput RNA interference (RNAi) Target sequencing (RIT-seq)

124 screen to identify cell cycle controls and regulators at a genomic scale. Key features of RIT-

125 seq screening include: first, use of a high-complexity T. brucei RNAi library comprising, in

126 this case, approximately one million clones; second, massive parallel tetracycline-inducible

127 expression of cognate dsRNA; and third, deep sequencing, mapping and counting of

128 mapped reads derived from RNAi target fragments (22). Each clone in the library has one of

129 approximately 100,000 different RNAi target fragments (250-1500 bp) between head-to-head

130 inducible T7-phage promoters, with each cassette inserted at a chromosomal locus that

131 supports robust expression. Inducibly expressed long dsRNA is then processed to siRNA by

132 the native RNAi machinery (27). Complexity and depth of genome coverage in the library are

133 critical, in that similar phenotypes produced by multiple RNAi target fragments against a

134 single gene provide cross-validation. Improvements in reference genome annotation (28),

135 next generation sequencing technology and sequence data analysis tools (see Materials and

136 Methods) have also greatly facilitated quantitative phenotypic analysis using short-read

137 sequence data.

138 Briefly, we induced massive parallel knockdown in an asynchronous T. brucei

139 bloodstream form RNAi library for $24 \mathrm{~h}$, fixed the cells, stained their DNA with propidium

140 iodide (PI) and then used high-speed fluorescence-activated cell sorting (FACS) to divide the

141 perturbed cell population into; sub-diploid ( $<2 \mathrm{C}), \mathrm{G}_{1}(2 \mathrm{C})$, S (between $2 \mathrm{C}$ and $\left.4 \mathrm{C}\right), \mathrm{G}_{2} \mathrm{M}(4 \mathrm{C})$

142 and over-replicated ( $>4 \mathrm{C}$ ) pools (Figure 1-figure supplement 1). Fixation and staining

143 with the fluorescent DNA intercalating dye were pre-optimised for high-speed sorting (see

144 Materials and Methods). Approximately 10 million cells were collected for each of the $G_{1}, S$

145 and $\mathrm{G}_{2} \mathrm{M}$ pools and samples from these pools were checked post-sorting to assess their

146 purity (Figure 1B, Figure 1-figure supplement 1). For the perturbed and less abundant 
Figure 1
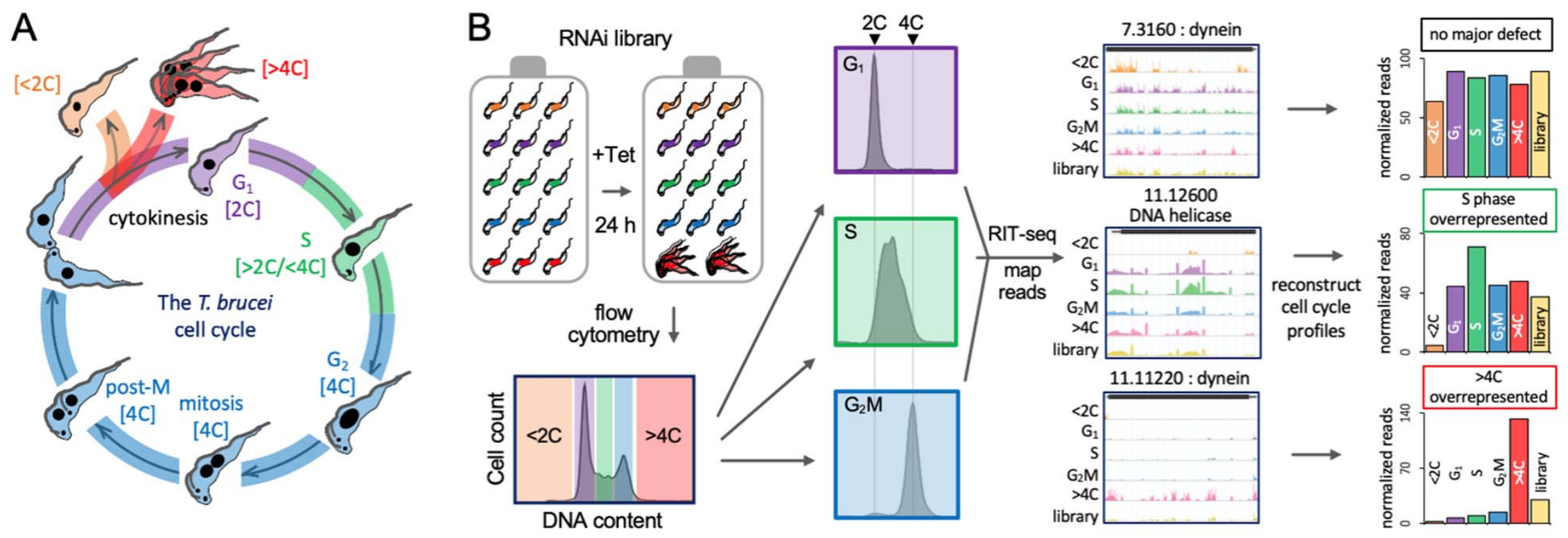

Figure 1. A genome-wide conditional knockdown screen for cell cycle progression defects. (A) Schematic representation of the bloodstream form $T$. brucei cell cycle, also showing aberrant sub-2C and $>4 \mathrm{C}$ phenotypes. (B) The schematic illustrates the RITseq screen; massive parallel induction of RNAi followed by flow cytometry and RIT-seq, allowing for reconstruction of cell cycle profiles, using mapped reads from each knockdown. Each read-mapping profile encompasses the gene of interest and associated untranslated regions present in the cognate mRNA. The library data represents the uninduced and unsorted population. GenelDs, Tb927.7.3160 for example, are shown without the common 'Tb927.' component. 
$147<2 \mathrm{C}$ and $>4 \mathrm{C}$ pools, less than one million cells were collected; these pools were retained in 148 their entirety for RIT-seq analysis.

149 RIT-seq was carried out for both the uninduced and induced, unsorted library

150 controls, and for each of the five induced and sorted pools of cells as described in the

151 Materials and Methods section. Briefly, we extracted genomic DNA from each sample,

152 amplified DNA fragments containing each RNAi target fragment in PCR reactions (Figure

153 1-figure supplement 1) and used the amplified products to generate Illumina sequencing

154 libraries. Analysis of sequencing reads mapped to the reference genome yielded counts for

155 both total reads as well as reads containing the barcode (GTGAGGCCTCGCGA) that flanks

156 each RNAi target fragment; the presence of the barcode confirmed that reads were derived

157 from a specific RNAi target fragment and not from elsewhere in the genome. We derived

158 counts of reads mapped to each of $>7,200$ non-redundant gene sequences in the uninduced

159 and induced, unsorted library controls and in each of the five sorted samples. We selected

160 the $24 \mathrm{~h}$ timepoint, equivalent to approximately 3.5 population doubling times, to allow

161 sufficient time for the development of robust inducible phenotypes, but also to capture

162 perturbed populations before they were critically diminished. Indeed, reads for $23.4 \%$ of

163 genes dropped by $>3$-fold following $72 \mathrm{~h}$ of knockdown in a prior RIT-seq study (23), while a

164 comparison of the unsorted control samples indicated that reads for only $0.6 \%$ of genes

165 dropped by >3-fold following $24 \mathrm{~h}$ of knockdown here (see Figure 1-figure supplement 2).

166 Each sorted-sample library yielded between 22.6 and 37 million mapped read-pairs; $<2 \mathrm{C}=$

$16737 \mathrm{M}, \mathrm{G}_{1}=35.1 \mathrm{M}, \mathrm{S}=30.3 \mathrm{M}, \mathrm{G}_{2} \mathrm{M}=22.6 \mathrm{M},>4 \mathrm{C}=24.9 \mathrm{M}$; yielding >35,000 RNAi data-

168 points (Supplementary File 1).

169 The RIT-seq digital data for individual genes following knockdown provided a

170 measure of abundance in each pool and were, therefore, used to digitally reconstruct cell

171 cycle profiles for individual gene knockdowns (Figure 1B). We expected to observe

172 accumulation of particular knockdowns in specific cell cycle phase pools, thereby reflecting

173 specific defects. This was indeed the case, and some examples are shown to illustrate; no

174 major defect, S phase overrepresented or $>4 \mathrm{C}$ overrepresented, following knockdown

175 (Figure 1B). These outputs suggest that loss of the cytoplasmic dynein heavy chain

176 (Tb927.7.3160) does not perturb cell cycle distribution; that a putative DNA helicase

177 (Tb927.11.12600) is required for the completion of S phase; and that knockdown of the

178 axonemal dynein heavy chain (Tb927.11.11220) results in endoreduplication in the absence

179 of cytokinesis. Dyneins are cytoskeletal motor proteins that move along microtubules, to

180 produce a flagellar beat, for example (29).

181

182 Validation and identification of $>\mathbf{1 , 0 0 0}$ candidates linked to cell cycle defects 
The $T$. brucei core genome comprises a non-redundant set of over 7,200 proteincoding sequences, for which we were now able to digitally reconstruct cell cycle profiles following knockdown; the full dataset is shown mapped to the eleven megabase chromosomes in Figure 2-figure supplement 1. The data can also be searched and browsed using an interactive, open access, online data visualization tool (see Figure 2figure supplement 2; https://tryp-cycle.onrender.com). First, we examined knockdowns reporting an overrepresentation of $>4 \mathrm{C}$ cells, indicating endoreduplication, and this yielded 201 genes for which reads in the $>4 C$ pool exceeded 1.5 -fold the sum of reads in the $G_{1}, S$ phase and $\mathrm{G}_{2} \mathrm{M}$ pools (Figure $2 \mathrm{~A}$, left-hand panel; Supplementary File 1 ). The $>4 \mathrm{C}$ phenotype was previously observed following $\alpha$-tubulin knockdown in a landmark study that first described RNAi in T. brucei (24) and, indeed, we observed pronounced overrepresentation of $>4 \mathrm{C}$ cells for both adjacent $\alpha$-tubulin (Tb927.1.2340) and $\beta$-tubulin (Tb927.1.2350) gene knockdowns (Figure 2A, middle and right-hand panel). We then examined knockdowns reporting an overrepresentation of $<2 \mathrm{C}$ cells, indicating a reduced DNA content. This yielded 119 genes for which reads in the $<2 \mathrm{C}$ pool exceeded 1.5 -fold the sum of reads in the $G_{1}, S$ phase and $G_{2} M$ pools (Figure $2 B$, left-hand panel; Supplementary File 1). Haploid cells were previously observed following DOT1A knockdown (30) and, consistent with the previous report, we observed pronounced overrepresentation of $<2 \mathrm{C}$ cells for the DOT1A (Tb927.8.1920) gene knockdown (Figure 2A, middle and right-hand panel); we are not aware of other knockdowns reported to yield a similar phenotype. Together, these results provide initial validation for the $>4 \mathrm{C}$ and $<2 \mathrm{C}$ components of the screen.

Next, we turned our attention to knockdowns reporting an overrepresentation of $\mathrm{G}_{1}, \mathrm{~S}$ phase or $\mathrm{G}_{2} \mathrm{M}$ cells. The pools of knockdowns that registered $>25 \%$ overrepresented read counts in each of these categories are highlighted in Figure 2C (left-hand panels and Supplementary File 1) and data for an example from each category are shown (Figure 2C; right-hand panels); the glycolytic enzyme, aldolase (Tb927.10.5620), reported 104\% increase in $\mathrm{G}_{1}$ cells (further details below); the proliferating cell nuclear antigen (PCNA; Tb927.9.5190), a DNA sliding clamp that is a central component of the replication machinery (31), reported 25\% increase in S phase cells, consistent with prior analysis (32); and PrimPol-like 2 (PPL2; Tb927.10.2520), a post-replication translesion polymerase, reported $65 \%$ increase in $\mathrm{G}_{2} \mathrm{M}$ cells, also consistent with prior analysis (33). These results provided initial validation for the $G_{1}, S$ phase and $G_{2} M$ components of the screen. cycle defect, based on the thresholds applied above. This is $16.1 \%$ of the 7,204 genes analysed, and the distribution of these genes among the five arms of the screen are shown 
Figure 2

A

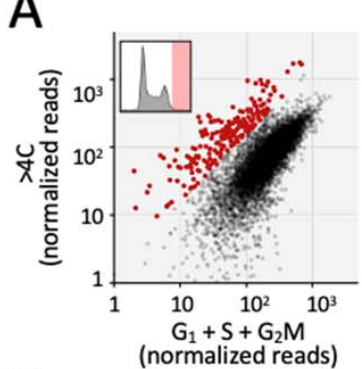

B

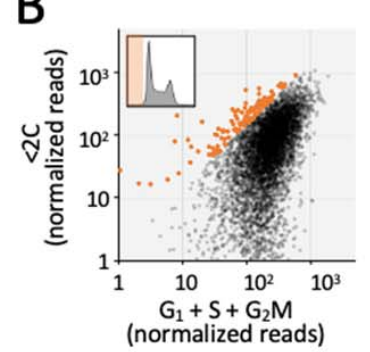

D

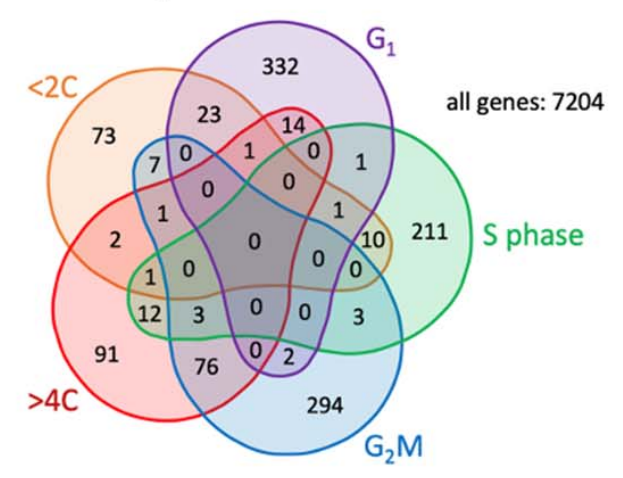

$\alpha / \beta$ tubulin:

DOT1A: 8.1920
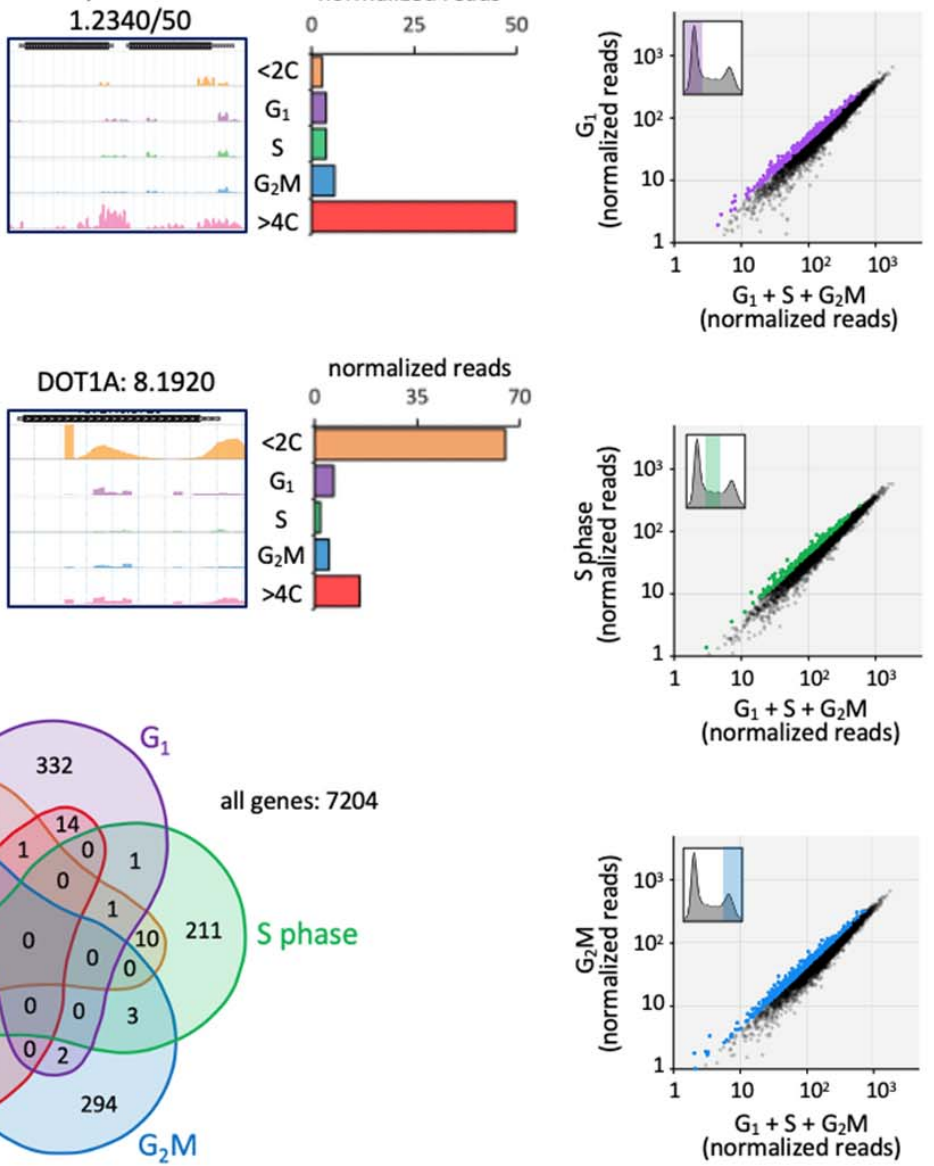

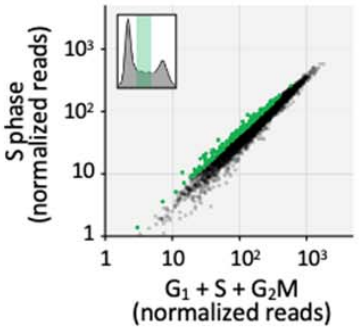

PCNA: 9.5190

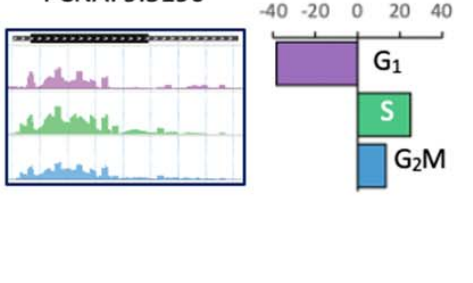

aldolase: 10.5620

change (\%)
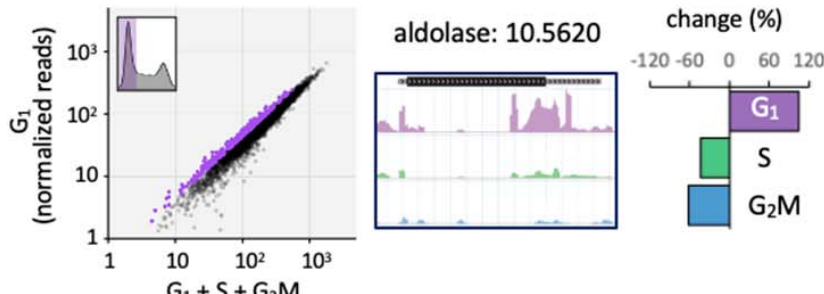

PPL2: 10.2520

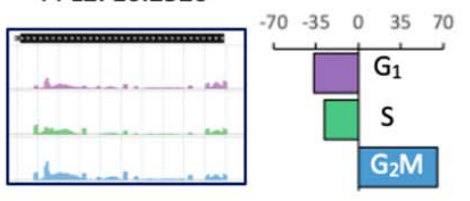

Figure 2. Validation and identification of $>1000$ candidates linked to cell cycle defects. (A) The plot on the left shows knockdowns overrepresented in the $>4 \mathrm{C}$ experiment in red; those with $>1.5$-fold the sum of reads in the $\mathrm{G}_{1}, \mathrm{~S}$ phase and $\mathrm{G}_{2} \mathrm{M}$ samples combined. The read-mapping profile and read-counts for $\alpha / \beta$-tubulin are shown to the right. (B) The plot on the left shows knockdowns overrepresented in the sub-2C experiment in orange; those with $>1.5$-fold the sum of reads in the $G_{1}, S$ phase and $G_{2} M$ samples combined. The readmapping profile and read-counts for DOT1A are shown to the right. (C) The plots on the left shows knockdowns overrepresented in the $G_{1}$, $S$ phase and $G_{2} M$ experiments in purple, green and blue, respectively; those that were $>25 \%$ overrepresented in each category. Read-mapping profiles and relative read-counts for example hits are shown to the right. PCNA, proliferating cell nuclear antigen; PPL2, PrimPol-like 2. (D) The Venn diagram shows the distribution of knockdowns overrepresented in each arm of the screen. 
in the Venn diagram in Figure 2D. Since we predicted that knockdowns associated with a cell cycle defect were more likely to also register a growth defect, we compared these datasets to prior RIT-seq fitness profiling data (23). All groups of genes that registered cell cycle defects, except for the $<2 \mathrm{C}$ set, were significantly enriched for genes that previously registered a loss-of-fitness phenotype following knockdown in bloodstream form cells $\left(\chi^{2}\right.$ test; $\left.<2 \mathrm{C}, p=0.15 ; \mathrm{G}_{1}, p=0.015 ; \mathrm{S}, p=4.7^{-4} ; \mathrm{G}_{2} \mathrm{M}, p=3.5^{-24} ;>4 \mathrm{C}, p=4.4^{-199}\right)$. This is consistent with loss-of-fitness as a common outcome following a cell cycle progression defect. Taken together, the analyses above provided validation for the RIT-seq based cell cycle phenotyping approach and yielded $>1,000$ candidate genes that impact specific steps during $T$. brucei cell cycle progression.

\section{Cytokinesis defects associated with endoreduplication}

In bloodstream form $T$. brucei, defective $>4 \mathrm{C}$ cells can arise due to endoreduplication without cytokinesis, either with $(24)$ or without $(25,26)$ mitosis. Cytokinesis-only defects were previously observed following knockdown of $\alpha$-tubulin (24) or flagellar proteins $(7,34)$; flagellar beat is thought to be required for cytokinesis in bloodstream form T. brucei. Consistent with these observations, $\alpha$-tubulin (see Figure 2A) and axonemal dynein heavy chain (see Figure 1B) knockdowns were amongst 201 genes overrepresented in the $>4 C$ pool in our screen. Gene Ontology (GO) annotations provide structured descriptions of gene products in terms of functions, processes, and compartments. Analysis of overrepresented GO annotations within the $>4$ C-enriched cohort revealed 'dynein', 'intraflagellar transport' (IFT), 'axoneme' and 'cytoskeleton' terms, and also 'chaperonin T-complex', 'cytokinesis' and multiple other 'cell cycle' terms (Figure 3A). The violin plot in Figure 3B shows specific enrichment of IFT and dynein knockdowns in the $>4 \mathrm{C}$ pool, relative to other cohorts of knockdowns. Exocyst components, primarily involved in exocytosis (35), were included as a negative control cohort; indeed, none of the exocyst components register enrichment in the $>4 \mathrm{C}$ pool, nor in any other pool (see below). Enrichment of individual chaperonin T-complex components, dyneins, and IFT factors in the $>4 \mathrm{C}$ pool is illustrated in Figure $3 \mathrm{C}$. The chaperonin T-complex is involved in tubulin and actin folding (36) and, notably, actin knockdown was also associated with $>4 \mathrm{C}$ enrichment (Figure 3-figure supplement 1 ).

The heat-map in Figure 3D shows the data for all five sorted pools for the cohorts described above and for additional cohorts of knockdowns enriched in the $>4 \mathrm{C}$ pool; these include radial spoke proteins, extra-axonemal paraflagellar rod (PFR) proteins, as well as nucleoporins. The gallery in Figure 3E shows examples of RIT-seq read-mapping profiles for twenty-four individual genes that register $>4 \mathrm{C}$ enrichment following knockdown. In 
A

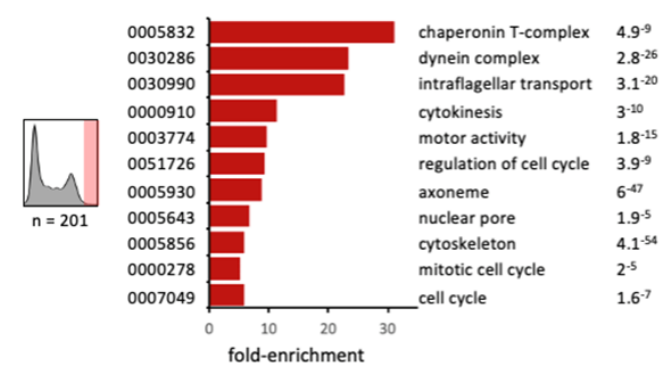

B

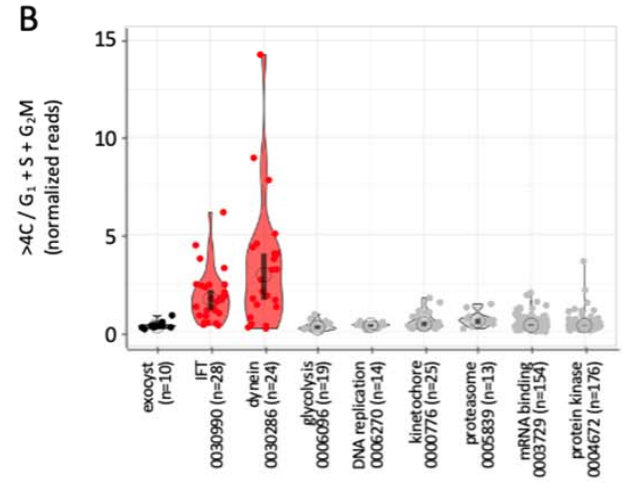

C

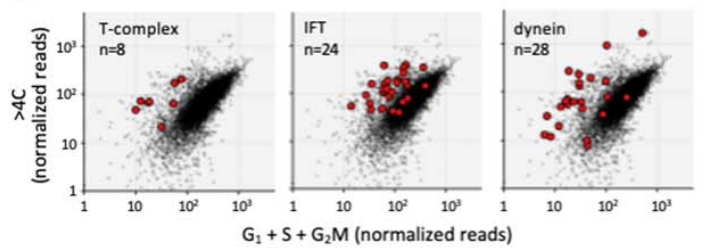

D

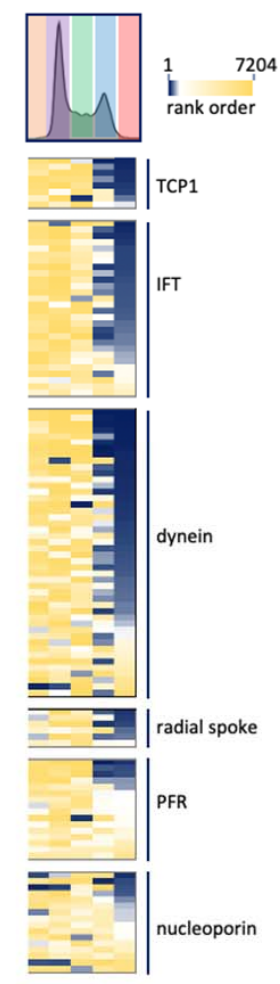

E

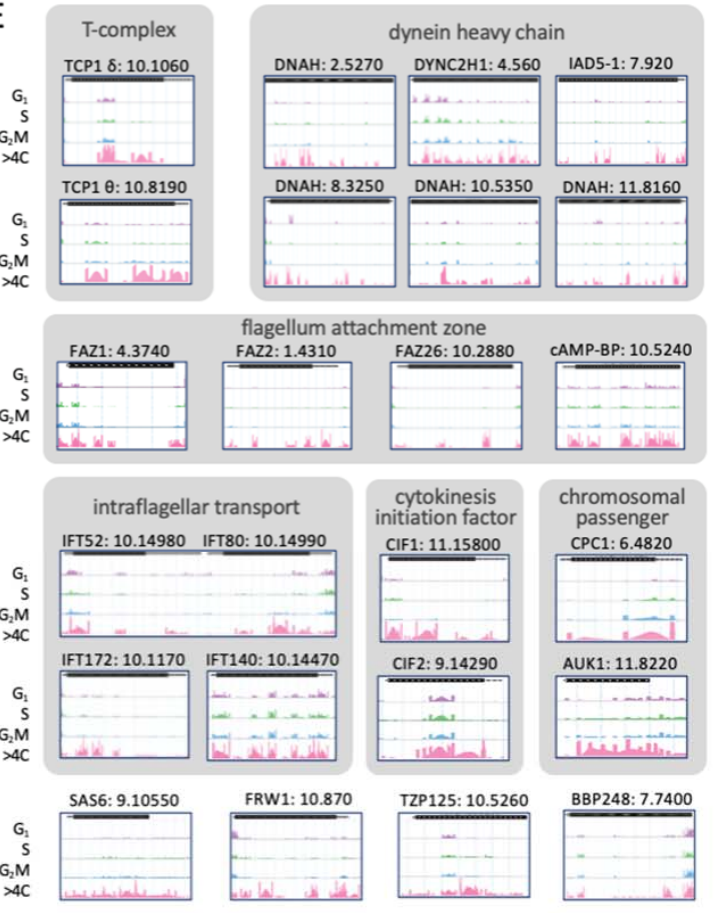

F ciliopathy-associated hits

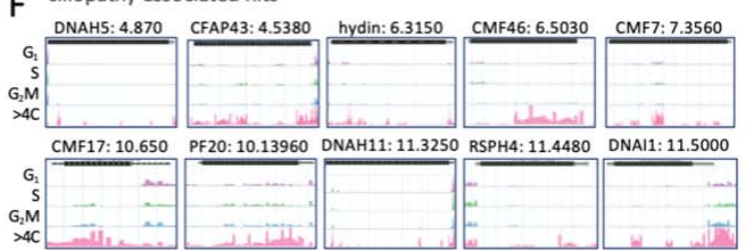

Figure 3. Cytokinesis defects associated with endoreduplication. (A) The bar-graph shows enriched Gene Ontology terms in the $>4 \mathrm{C}$ overrepresented dataset. (B) The violin plot shows relative $>4 \mathrm{C}$ read-counts for cohorts of genes and reflects data distribution. Open circles indicate median values and the vertical bars indicate $95 \%$ confidence intervals. Significantly overrepresented cohorts are indicated in red. (C) The plots show overrepresentation of T-complex, dynein and intraflagellar transport (IFT) factors in red in the >4C experiment. (D) The heatmaps show relative representation in all five sorted pools for the above and additional cohorts of knockdowns; blue, most overrepresented. (E) Example read-mapping profiles for hits overrepresented in the $>4 \mathrm{C}$ pool. (F) Example read-mapping profiles for ciliopathy-associated hits overrepresented in the $>4 \mathrm{C}$ pool. CMF, Component of Motile Flagella; CFAP, Cilia and Flagella Associated Protein. 
which mediate attachment of the flagellum to the cell body (38); cytokinesis initiation factors CIF1/TOEFAZ1 (Tb927.11.15800) and CIF2 (Tb927.9.14290) (39) and chromosomal passenger complex components, including CPC1 (Tb927.6.4820) and the aurora B kinase, AUK1 (Tb927.11.8220). AUK1 and CPC1 are spindle-associated and regulate mitosis and cytokinesis $(26,40)$. Notably, endoreduplication was reported previously following AUK1 knockdown in bloodstream form T. brucei (41) and this is the kinase with the most pronounced overrepresentation in our $>4 \mathrm{C}$ dataset. The next $>4 \mathrm{C}$ overrepresented kinase is the CMGC/RCK (Tb927.3.690), knockdown of which previously yielded a dramatic cytokinesis defect (42).

Additional examples of genes registering $>4 \mathrm{C}$ overrepresentation include the centriole cartwheel protein SAS6 (Tb927.9.10550) (43), the cleavage furrow-localizing protein FRW1 (Tb927.10.870) (44), the basal body - axoneme transition zone protein TZP125 (Tb927.10.5260) (45) and the basal body protein BBP248 (Tb927.7.7400) (46). One hundred additional examples are shown in Figure 3-figure supplement 1, including intermediate and light chain dyneins; other flagellum-associated factors, radial spoke proteins, components of motile flagella, flagellum attachment and transition zone proteins, intraflagellar transport proteins, kinesins $(47,48)$, nucleoporins $(49)$, and many previously uncharacterised hypothetical proteins. Some other notable examples include the microtubule-severing katanin KAT80 (Tb927.9.9960) (50), the dynein regulatory factor trypanin (Tb927.10.6350) (51), the AIR9 microtubule associated protein (Tb927.11.17000) (52), CAP51V (Tb927.7.2650) (53) and importin, IMP1 (Tb927.9.13520) (54).

Orthologues of several T. brucei flagellar proteins have previously been linked to debilitating human ciliopathies, such that the trypanosome flagellum is used as a model for studies on these defects. Defects in intraflagellar dynein transport are associated with respiratory infections, for example (9). Some examples of ciliopathy-associated orthologues which register overrepresentation in the $>4 \mathrm{C}$ pool are shown in Figure $3 F$ and Figure 3 figure supplement 1 . These include proteins linked to primary ciliary dyskinesia (DNAI1, Tb927.11.5000; DNAH5, Tb927.4.870; DNAH11, Tb927.11.3250; RSPH4, Tb927.11.4480) (11); male infertility (CFAP43, Tb927.4.5380; CMF7/TbCFAP44, Tb927.7.3560) (10); and cone-rod dystrophies, as well as other ocular defects (CMF17, Tb927.10.650; CMF39, Tb927.4.5370; CMF46, Tb927.6.5030) (8).

From analysis of knockdowns overrepresented in the $>4 \mathrm{C}$ pool, we conclude that RIT-seq screening provided comprehensive genome-scale identification of cytokinesis defects in bloodstream form T. brucei. Endoreduplication appears to be a common outcome following a cytokinesis defect. Amongst hundreds of genes required for progression through cytokinesis, flagellar proteins featured prominently, including the majority of dynein chains and intraflagellar transport factors. Many of these factors are essential for viability and 
Figure 4

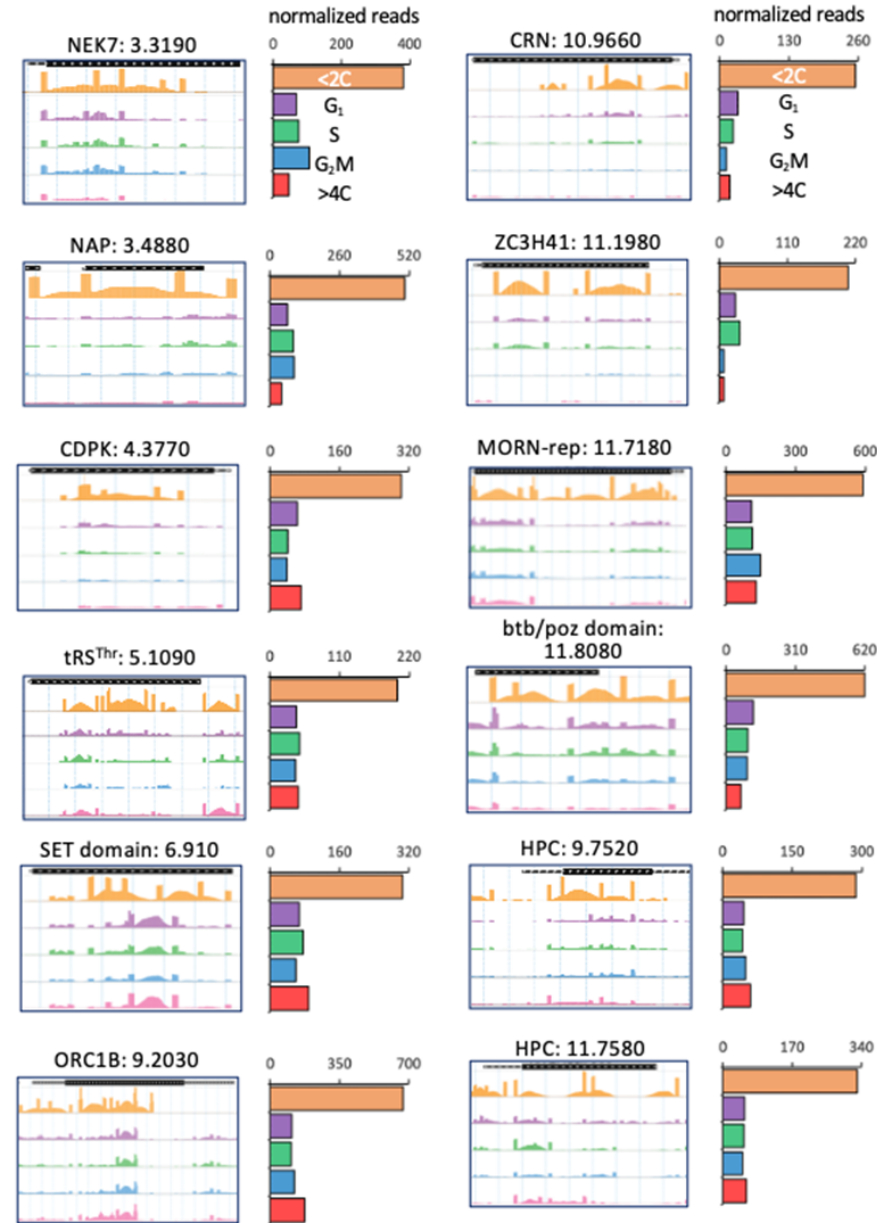

Figure 4. Defects producing sub-diploid cells. Read-mapping profiles and read-counts for example hits overrepresented in the $<2 \mathrm{C}$ experiment. HPC, Hypothetical Protein, Conserved. 
292 include potential druggable targets in trypanosomatids, as well as orthologues of proteins

293 associated with ciliopathies.

\section{Defects producing sub-diploid cells}

296 A DNA replication or mitosis defect followed by cytokinesis may result in generation of cells that retain nuclear DNA with a sub-2C DNA content. We emphasise retention of nuclear DNA here because $T$. brucei cells lacking nuclear DNA, referred to as zoids, have been reported previously as a result of asymmetrical cell division. Zoids are typically observed when DNA replication or mitosis are perturbed in insect stage cells $(16,25,55)$. The zoid phenotype is typically either absent or less abundant in the developmentally distinct bloodstream form cells (56) that we analysed here. Nevertheless, any zoids present in the $<2 \mathrm{C}$ pool will not have been detected using RIT-seq, since detection relies upon the presence of a nuclear RNAi target fragment.

One hundred and nineteen knockdowns were overrepresented in the $<2$ C RIT-seq screening dataset. A particularly prominent hit was the previously identified histone methyltransferase, DOT1A (see above). DOT1A is responsible for dimethylation of histone H3K76, and DOT1A knockdown results in premature mitosis without DNA replication, generating cells with a haploid DNA content (30). The gallery in Figure 4 shows examples of RIT-seq read-mapping profiles for twelve additional genes that register $<2 \mathrm{C}$ enrichment following knockdown. These include the DNA replication origin recognition complexassociated protein, ORC1B (Tb927.9.2030) (57), two putative protein kinases (NEK7, Tb927.3.3190; CDPK, Tb927.4.3770), a putative mRNA-binding helicase (ZC3H41,

314 Tb927.11.1980), a nucleosome assembly protein (NAP, Tb927.3.4880) and threonyl-tRNA

315 synthetase (Tb927.5.1090). Notably, threonine metabolism impacts histone methylation in mammalian cells (58), while a protein containing a putative methyllysine-binding SET domain (Tb927.6.910) also registers enrichment in the $<2 \mathrm{C}$ pool. These hits present new candidate regulators of DNA replication, mitosis or meiosis (59), and further potential links to post-translational protein methylation as a key player in coordinating these processes.

\section{A profile of $G_{1}, S$ phase and $G_{2} M$ defects}

We next analysed knockdowns overrepresented in the $G_{1}$, S phase or $G_{2} M$ pools. Several hundred knockdowns registered $>25 \%$ overrepresented read counts in each of these categories, as shown in a RadViz plot (Figure 5A). GO annotations within each cohort revealed a number of enriched terms (Figure 5B). Overrepresented knockdowns were associated with glycolysis and mRNA binding in the $G_{1}$ pool, with DNA replication in the $S$ phase pool and with a similar profile to that seen for the $>4 \mathrm{C}$ set in the $\mathrm{G}_{2} \mathrm{M}$ pool. 
Figure 5
A

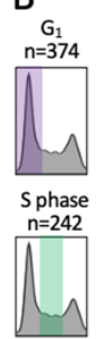

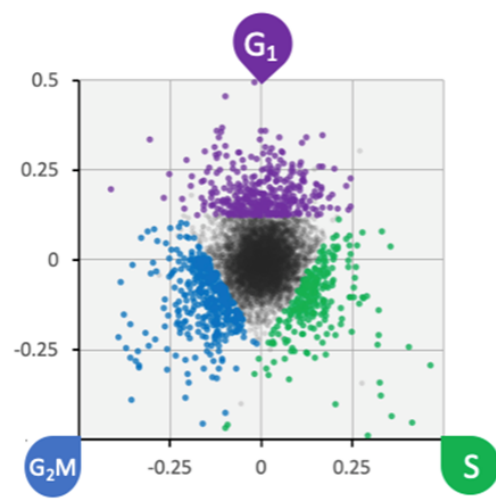

C
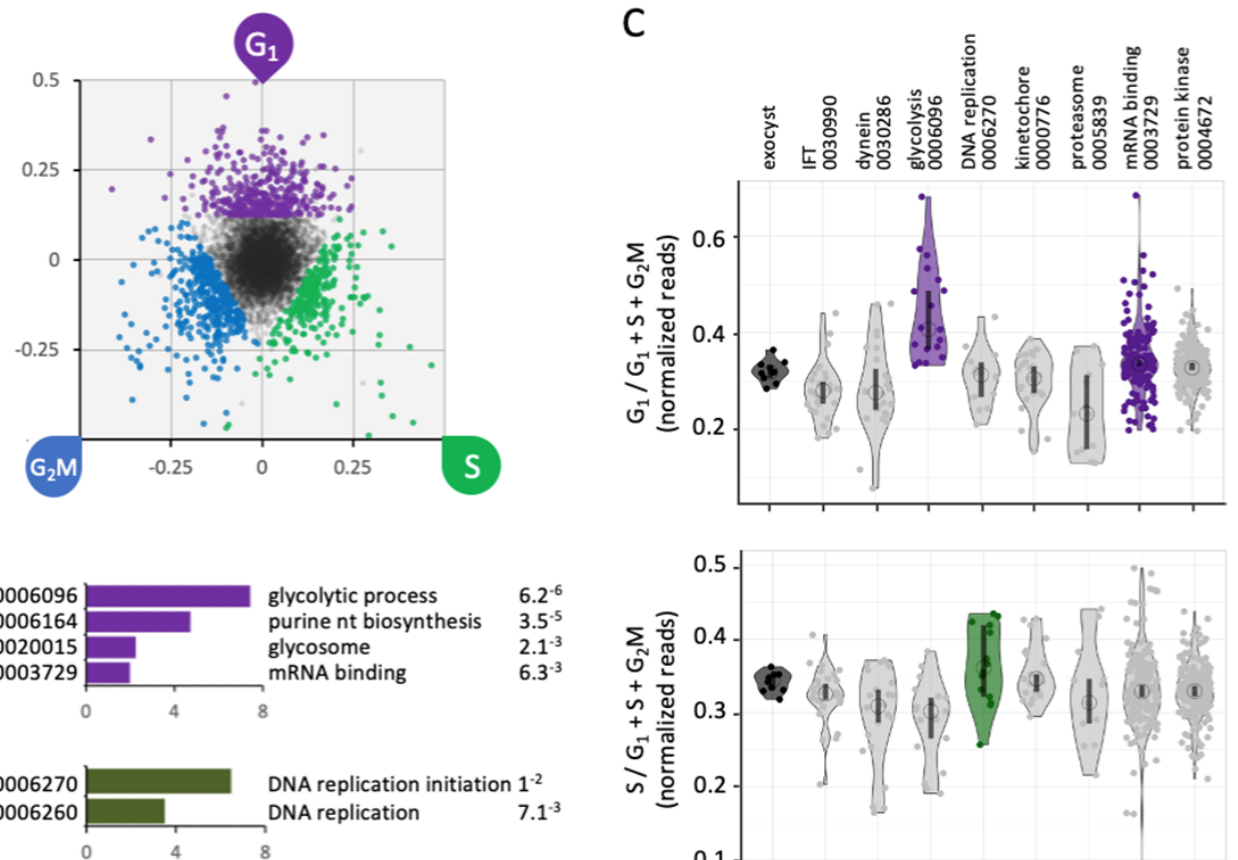

0006270 DNA
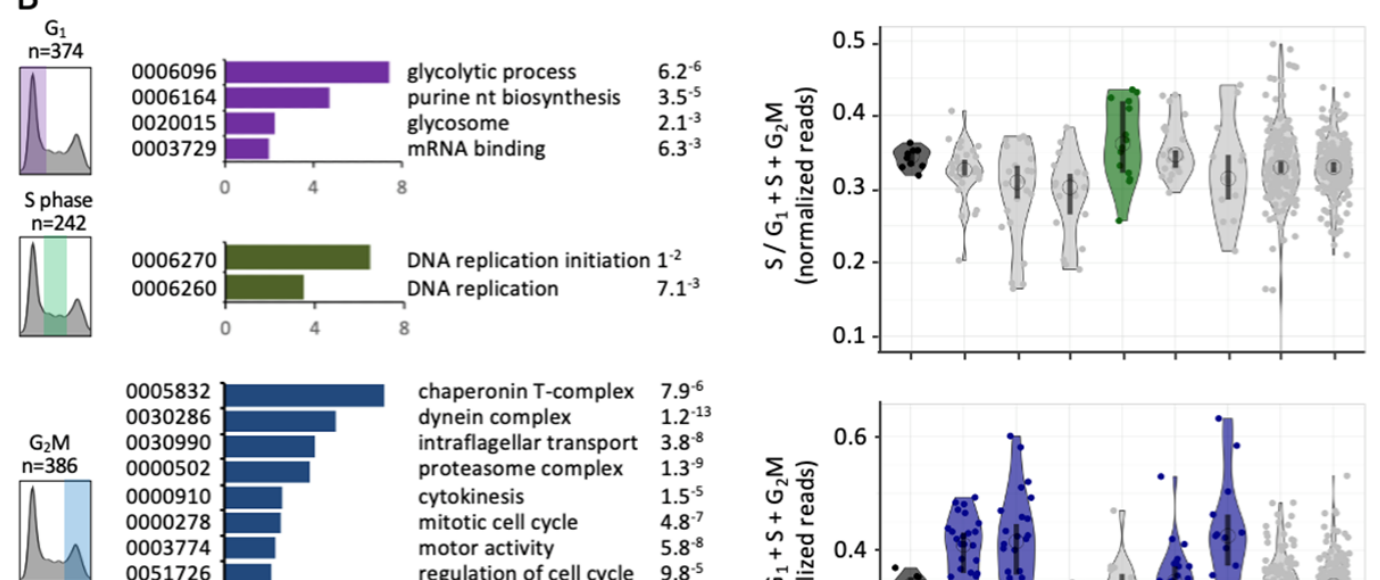

0000502

0000910
0000278

0003774

0051726

0007049
0005643

0005930

0005856

${ }^{0} \stackrel{8}{8}{ }^{16}$ intraflagellar transport $3.8^{-8}$ proteasome complex cytokinesis mitotic cell cycle motor activity regulation of cell cycle $\quad 9.8^{-5}$ axoneme axoneme
cytoskeleto

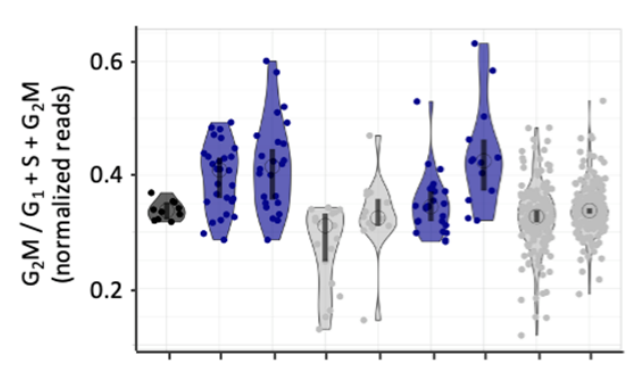

D
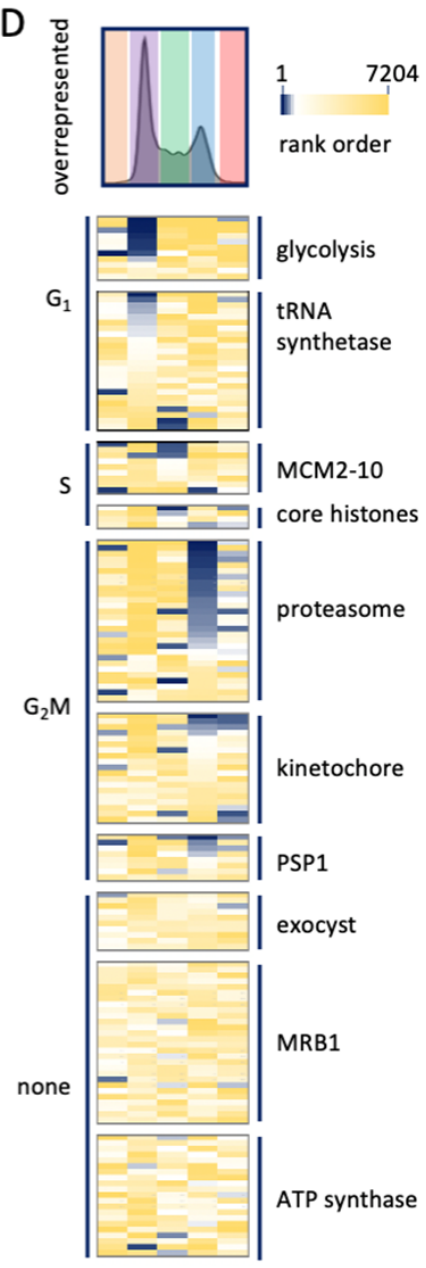

Figure 5. A profile of $G_{1}, S$ phase and $G_{2} /$ mitosis defects. (A) The RadViz plot shows knockdowns that registered $>25 \%$ overrepresented read-counts in the $G_{1}$, $S$ phase or $G_{2} M$ categories. (B) The bar-graphs shows enriched Gene Ontology terms in the $G_{1}$, S phase or $G_{2} M$ overrepresented datasets. (C) The violin plots show relative $G_{1}$, S phase or $G_{2} M$ read-counts for cohorts of genes and reflects data distribution. Open circles indicate median values and the vertical bars indicate 95\% confidence intervals. Overrepresented cohorts are indicated in purple, green and blue, respectively. (D) The heatmaps show relative representation in all five sorted pools for the above and additional cohorts of knockdowns; blue, most overrepresented. 
The violin plot in Figure 5C shows specific enrichment of individual knockdowns for glycolytic enzymes and a subset of mRNA binding protein in the $\mathrm{G}_{1}$ pool, for DNA replication factors in the S phase pool, and proteasome components and a subset of kinetochore component in the $\mathrm{G}_{2} \mathrm{M}$ pool (Figure $5 \mathrm{C}$, lower panel). Overlap between knockdowns that accumulate in both the $\mathrm{G}_{2} \mathrm{M}$ and $>4 \mathrm{C}$ pools likely reflects mitosis and cytokinesis defects both before and after endoreduplication; perhaps $24 \mathrm{~h}$ of knockdown is insufficient for endoreduplication in all perturbed cells or not all mitosis or cytokinesis-perturbed phenotypes result in endoreduplication; compare $>4 \mathrm{C}$ and $\mathrm{G}_{2} \mathrm{M}$ data for IFT factors and dyneins in Figure $3 B$ and Figure $5 C$, for example. Once again, exocyst components provided a control cohort with no components registering enrichment in the $G_{1}, S$ phase or $G_{2} M$ pools following knockdown (Figure 5C).

The heat-map in Figure 5D shows the data for all five sorted pools for the cohorts described above and for additional knockdowns enriched in the $G_{1}$ or $S$ phase (tRNA synthetases), S phase (core histones) or $\mathrm{G}_{2} \mathrm{M}$ pools (PSP1, DNA polymerase suppressor 1), or not enriched in any pool. These latter sets provide further controls that do not appear to have specific impacts on cell cycle progression, including the mitochondria RNA editing accessory complex MRB1 (60) and the mitochondrial ATP synthase complex $V(61)$. Thus, we identify a number of protein complexes, pathways and regulatory factors that are specifically required for progressive steps through the trypanosome cell cycle.

\section{Pathways and protein complexes associated with $G_{1}, S$ phase and $G_{2} M$ defects}

We next explored some of the cohorts of hits described above in more detail. Glycolytic enzymes are particularly prominent amongst knockdowns that accumulate in $\mathrm{G}_{1}$ and we illustrate the RIT-seq profiling data for these enzymes in Figure 6A. Seven of eleven glycolytic enzyme knockdowns register $>25 \%$ overrepresentation in the $\mathrm{G}_{1}$ pool; hexokinase (Tb927.10.2010), phosphofructokinase (Tb927.3.3270), aldolase (see Figure 2C), triosephosphate isomerase (Tb927.11.5520), glyceraldehyde 3-phosphate dehydrogenase (Tb927.6.4280), phosphoglycerate kinase C (Tb927.1.700) and pyruvate kinase (Tb927.10.14140). Glycolysis operates in peroxisome-like organelles known as glycosomes in trypanosomes and is thought to be the single source of ATP in bloodstream form cells (12). Glycolysis also provides metabolic intermediates that support nucleotide production. Notably, mammalian cell proliferation is accompanied by activation of glycolysis, and the Warburg effect relates to this phenomenon in oncology (62). Indeed, hexokinase regulates the $G_{1} / S$ checkpoint in tumour cells (63). The results are also consistent with the observation that $T$. brucei accumulate in $\mathrm{G}_{1}$ or $\mathrm{G}_{0}$ under growth-limiting conditions (64) or during differentiation to the non-dividing stumpy form (65), possibly reflecting a role for glucose 
Figure 6
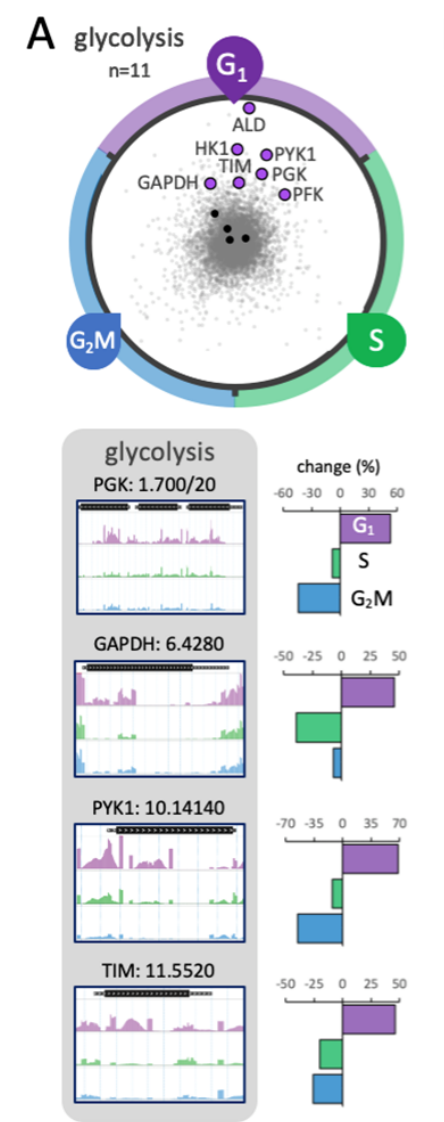

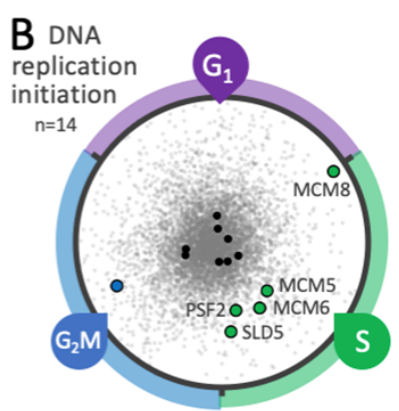

DNA replication SLDS (GINS): 3.4810 change (\%)

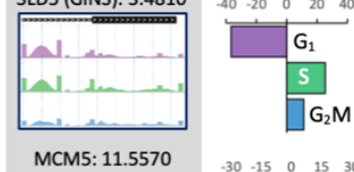

MCM5: 11.5570
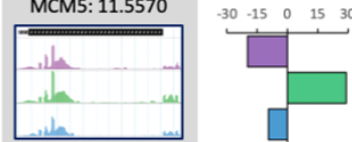

PSF2 (GINS): 11.10460

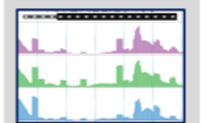

MCM6: 11.11640
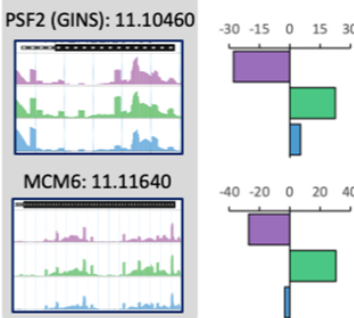
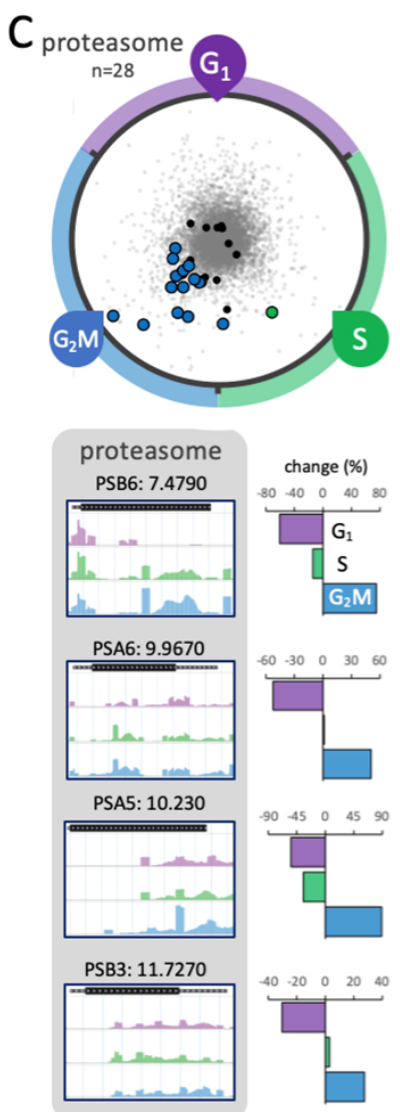

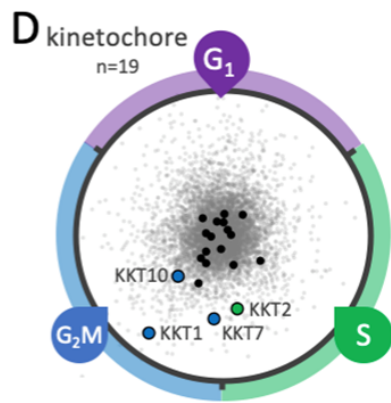

kinetochore
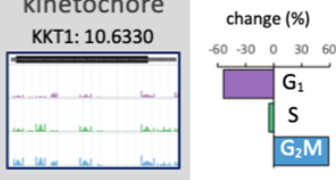

KKT2: 11.10520

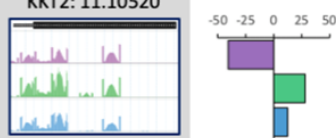

KKT7: 11.1030
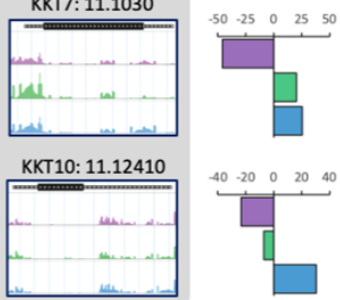

Figure 6. Protein complexes and pathways associated with $\mathrm{G}_{1}, \mathrm{~S}$ phase and $\mathrm{G}_{2}$ /mitosis defects. (A) The RadViz plot shows glycolytic enzyme knockdowns. Those that registered $>25 \%$ overrepresented read-counts in the $\mathrm{G}_{1}$ category are indicated, purple. Black datapoints indicate other genes from each cohort. Grey data-points indicate all other genes. The read-mapping profiles and relative readcounts in the lower panel show example hits. (B) As in a but for DNA replication initiation factor knockdowns that registered $>25 \%$ overrepresented read-counts in the $\mathrm{S}$ phase category, indicated in green. (C) As in a but for proteasome component knockdowns that registered $>25 \%$ overrepresented read-counts in the $\mathrm{G}_{2} \mathrm{M}$ category, indicated in blue. (D) As in a but for kinetochore component knockdowns that registered $>25 \%$ overrepresented read-counts in the $S$ phase or $G_{2} M$ categories, indicated in green or blue, respectively. 
in stumpy-form cells (67). We conclude that, as in other organisms (68), there is metabolic control of the cell cycle and a nutrient sensitive restriction point in $T$. brucei, with glycolysis playing a role in the $G_{1}$ to $S$ phase transition.

DNA replication initiation factors are particularly prominent amongst knockdowns that accumulate in S phase and we illustrate the RIT-seq profiling data for these factors in Figure $6 B$. Five knockdowns that register $>25 \%$ overrepresentation in the $S$ phase pool are components of the eukaryotic replicative helicase, the CMG (Cdc45-MCM-GINS) complex. At the core of this complex is the minichromosome maintenance complex (MCM2-7), a helicase that unwinds the duplex DNA ahead of the moving replication fork (69). Identification of this subset of components suggests that these particular subunits are limiting for progression through $\mathrm{S}$ phase.

Proteasome components are particularly prominent amongst knockdowns that accumulate in $\mathrm{G}_{2} \mathrm{M}$, and we illustrate the RIT-seq profiling data for this protein complex in Figure 6 C. Sixteen of 28 proteasome component knockdowns register $>25 \%$ overrepresentation in the $\mathrm{G}_{2} \mathrm{M}$ pool. This output suggests that the $T$. brucei proteasome is most likely responsible for degrading cell cycle regulators, such as poly-ubiquitinated cyclins, which are known to control cell cycle checkpoints in other eukaryotes. Candidate target cyclins in T. brucei include: cyclin 6 (CYC6, Tb927.11.16720), degradation of which is required for mitosis (70); cyclin-like CFB2 (Tb927.1.4650), required for cytokinesis (71); cyclin 2 (CYC2, Tb927.6.1460) or cyclin 3 (CYC3, Tb927.6.1460), which have short halflives and a candidate destruction box motif in the case of CYC3 (72).

Kinetochore components (17) are also amongst knockdowns that accumulate in $\mathrm{G}_{2} \mathrm{M}$ and we illustrate the RIT-seq profiling data for this protein complex in Figure 6D. Although knockdown of KKT2 (Tb927.10.10520), a putative kinase, registered overrepresentation in the S phase pool, KKT1 (Tb927.10.6330), KKT7 (Tb927.11.1030) and KKT10 (CLK1, Tb927.11.12410) knockdowns registered $>25 \%$ overrepresentation in the $\mathrm{G}_{2} \mathrm{M}$ pool, suggesting that these particular kinetochore components, which all display temporal patterns of phosphorylation from $S$ phase to $\mathrm{G}_{2} \mathrm{M}(21)$, are limiting for progression through mitosis. Notably, KKT10 is a kinase responsible for phosphorylation of KKT7, which is required for the metaphase to anaphase transition (73); as well as for the phosphorylation of KKT1, which is required for kinetochore assembly (74). These findings are consistent with the view that kinetochore components control a non-canonical spindle checkpoint in trypanosomes (73).

RBPs, kinases and hypothetical proteins associated with $G_{1}, S$ phase and $G_{2} M$ defects Widespread polycistronic transcription in trypanosomatids places great emphasis on post-transcriptional controls and, consistent with this, knockdowns overrepresented in the 

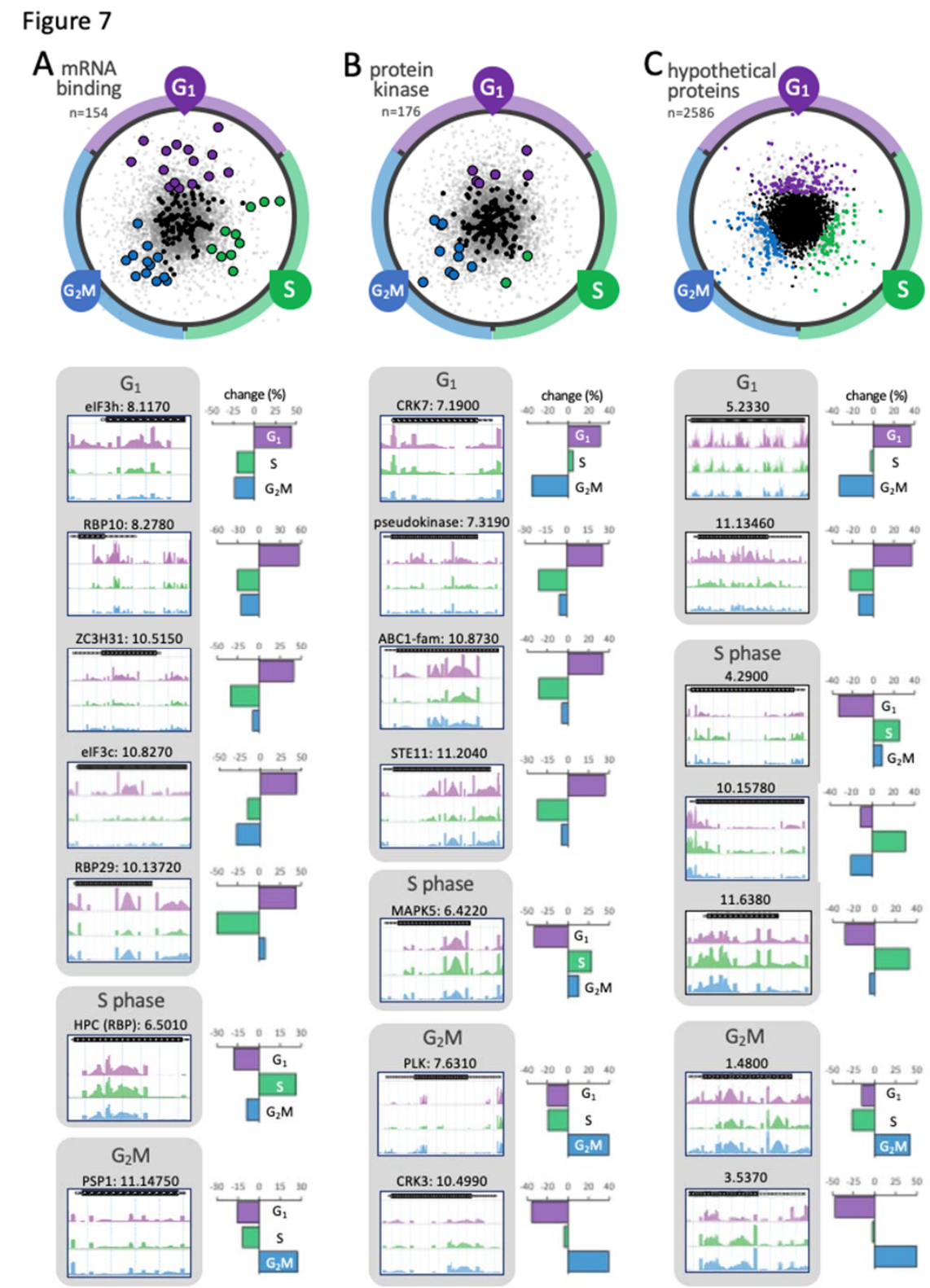

Figure 7. RBPs kinases and hypothetical proteins associated with gap and S phase defects. (A) The RadViz plot shows mRNA binding protein knockdowns (RBPs). Those that registered $>25 \%$ overrepresented read-counts in the $G_{1}, S$ phase or $G_{2} M$ categories are indicated, in purple, green and blue, respectively. The read-mapping profiles and relative read-counts in the lower panels show example hits. (B) As in a but for protein kinase knockdowns. (C) As in a but for hypothetical (conserved) protein knockdowns. 
$402 \mathrm{G}_{1}$, S phase and $\mathrm{G}_{2} \mathrm{M}$ pools revealed many putative mRNA binding proteins (RBPs) and

403 kinases. Indeed, RBPs are significantly enriched amongst knockdowns that registered $\mathrm{G}_{1}, \mathrm{~S}$

404 phase or $\mathrm{G}_{2} \mathrm{M}$ cell cycle defects $\left(\chi^{2}\right.$ test, $\left.p=7^{-5}\right)$. We show the RIT-seq profiling data for

405 seven RBP knockdowns that register $>25 \%$ overrepresentation in these pools (Figure $7 A$ ).

406 These include knockdowns for two components of the translation initiation factor, elF3,

407 linked to accumulation in $\mathrm{G}_{1}$ (Tb927.8.1170; Tb927.10.8270). Proposed regulatory functions

408 for elF3 include reinitiation of translation on polycistronic mRNAs and as a substrate for

409 translation regulatory kinases (75). The RBP10 (Tb927.8.2780), RBP29 (Tb927.10.13720)

410 and ZC3H31 (Tb927.10.5150) knockdowns were also enriched in $\mathrm{G}_{1}$. RBP10, in particular,

411 has been characterised in some detail and promotes the bloodstream form state (76). Thus,

412 the RIT-seq cell cycle screen implicated a number of specific RBPs in post-transcriptional

413 control of cell cycle progression, most likely through modulation of mRNA stability and/or

414 translation of cell cycle regulators.

415 We show data for several protein kinases above, linked to enriched $>4 C$ (Figure 3D),

416 sub-2C (Figure 4), S phase or $\mathrm{G}_{2} \mathrm{M}$ (Figure 6D) phenotypes, and now show the RIT-seq

417 profiling data for seven additional protein kinase knockdowns that register $>25 \%$

418 overrepresentation in the $\mathrm{G}_{1}, \mathrm{~S}$ phase or $\mathrm{G}_{2} \mathrm{M}$ pools (Figure $7 B$ ). These include knockdowns

419 for CRK7 (Tb927.7.1900), linked to accumulation in $\mathrm{G}_{1}$; MAPK5 (Tb927.6.4220), linked to

420 accumulation in S phase and polo-like kinase (PLK, Tb927.7.6310) and cdc2-related kinase

4213 (CRK3, Tb927.10.4990), linked to accumulation in $\mathrm{G}_{2} \mathrm{M}$. PLK was previously shown to

422 control cell morphology, furrow ingression and cytokinesis (77-79), while CRK3 was shown

423 to play a role in $\mathrm{G}_{2} \mathrm{M}$ progression in bloodstream form T. brucei $(42,80)$.

424 Finally, we analysed genes encoding proteins annotated as hypothetical (conserved).

425 Despite excellent progress in genome annotation, 35\% of the non-redundant gene-set in $T$.

426 brucei retain this annotation, amounting to $>2,500$ genes. We show data for more than

427 twenty of these knockdowns above, linked to enriched $>4 \mathrm{C}$ (Figure 3-figure supplement

428 1) or sub-2C (Figure 4) phenotypes, and we identify additional hypothetical (conserved)

429 protein knockdowns that register $>25 \%$ overrepresentation in the $G_{1}$, S phase or $G_{2} M$ pools

430 RIT-seq profiling data are shown for seven examples in Figure $7 C$ and for twenty four

431 examples in Figure 7 - figure supplement 1. Amongst fifty-four other examples of

432 knockdowns shown in Figure 7-figure supplement 1, are alternative oxidase

433 (Tb927.10.7090) (81), linked to $G_{1}$ enrichment; tRNA synthetases linked to $G_{1}$ (Trp,

434 Tb927.3.5580; His, Tb927.6.2060) or S phase enrichment (Glu, Tb927.6.4590; Lys,

435 Tb927.8.1600); kinesins linked to $S$ phase (Tb927.7.7120) or $\mathrm{G}_{2} \mathrm{M}$ enrichment, including

436 both chromosomal passenger complex kinesins (26) (Tb927.11.2880, KIN-A and

437 Tb927.7.5040, KIN-B) and KIN-G (Tb927.6.1770); CYC6 $(25,82)$, CFB2 (71), centrin 3

438 (Tb927.10.8710) (83) and, finally, both components of the histone chaperone FACT 
(facilitates chromatin transcription) complex (84) Spt16 (Tb927.3.5620) and Pob3 (Tb927.10.14390), linked to $\mathrm{G}_{2} \mathrm{M}$ enrichment. Notably, the FACT complex has been linked to centromere function in human cells (85). quantitative transcriptome (19), proteome (20) and phosphoproteome (21) cell cycle profiling data. An initial survey of all 1,158 genes that registered a cell cycle defect here (see Figure 2D) revealed significant enrichment of cell cycle regulated mRNAs (overlap $=105$ of 485, $\chi^{2}$ $p=8.5^{-4}$ ), as well as proteins displaying cell cycle regulated phosphorylation (overlap $=106$ of $\left.547, \chi^{2} p=0.035\right)$. It is important to note here, however, and for the analyses below, that these transcriptome and (phospho)proteome datasets were derived from insect stage $T$. brucei, meaning that regulation may differ in some cases in bloodstream $T$. brucei cells used for RIT-seq analysis here.

Specific transcripts required for cell cycle progression are likely upregulated prior to peak demand for the encoded protein, and we found evidence to support this view. For example, transcripts upregulated in late $G_{1}$ or in $S$ phase were enriched amongst those knockdowns linked to accumulation in the $\mathrm{G}_{2} \mathrm{M}$ pool here $\left(\chi^{2} p=3.3^{-3}\right.$ and $p=1.1^{-2}$ respectively); $G_{1}$ upregulated transcripts included, for example, both components of the FACT complex (see Figure 7-figure supplement 1 ). In addition, $S$ phase and $\mathrm{G}_{2} \mathrm{M}$ upregulated transcripts, including those encoding multiple flagellum-associated proteins, were enriched amongst knockdowns linked to accumulation in the $>4 \mathrm{C}$ pool $\left(\chi^{2} p=4.6^{-18}\right.$ and $p=2.4^{-5}$ respectively).

Although the overlap between cell cycle regulated proteins (20) and those knockdowns that registered a cell cycle defect here failed to achieve significance (overlap = 71 of $367, \chi^{2} p=0.09$ ), cell cycle regulated proteins do appear to be required for progression through specific stages of the cell cycle. For example, multiple glycolytic enzymes upregulated in $G_{1}$ were linked to accumulation in the $G_{1}$ pool following knockdown $\left(\chi^{2} p=\right.$ 7.9-11). In addition, proteins highly upregulated in $\mathrm{G}_{2}$ and $M$ were linked to accumulation in the $\mathrm{G}_{2} \mathrm{M}\left(\chi^{2} p=1.8^{-8}\right)$ or $>4 \mathrm{C}$ pools $\left(\chi^{2} p=8.9^{-9}\right)$ following knockdown, including multiple kinetochore and chromosomal passenger complex components, respectively.

In terms of specific cell cycle regulated genes/proteins, we focused on those that previously registered a significant loss of fitness following knockdown (23) and now with a

473 RIT-seq based cell cycle progression functional assignment. Examples include putative 
475 upregulation in $\mathrm{G}_{1}$, protein upregulation in $\mathrm{S}$ phase, cell cycle regulated phosphorylation and 476 accumulation in $\mathrm{G}_{2} \mathrm{M}$ following knockdown (see Figure $5 \mathrm{D}$ and Figure $7 A$ ). The kinetochore 477 components, KKT1 and KKT7, and also CRK3, all display mRNA upregulation in S phase,

478 protein upregulation in $\mathrm{G}_{2}$ and $\mathrm{M}$, cell cycle regulated phosphorylation and accumulation in

$479 \mathrm{G}_{2} \mathrm{M}$ following knockdown (see Figure 6D and Figure 7B); KKT10 and CYC6 report a

480 similar profile (see Figure 6D), except for the mRNA regulation component. The cytokinesis

481 initiation factors, CIF1 and CIF2, also display mRNA upregulation in S phase, protein

482 upregulation in $\mathrm{G}_{2}$ and $\mathrm{M}$ and cell cycle regulated phosphorylation, but instead accumulation

483 in the $>4 \mathrm{C}$ pool following knockdown (see Figure $3 E$ ). Finally, the chromosomal passenger

484 complex components, CPC1 and AUK1, as well as furrow localized FRW1, report mRNA

485 and protein upregulation in $\mathrm{G}_{2} \mathrm{M}$ and accumulation in the $>4 \mathrm{C}$ pool following knockdown (see

486 Figure $3 E$ ). Thus, several regulators linked to specific cell cycle progression defects by RIT-

487 seq profiling, are themselves regulated. 


\section{Discussion}

Despite intense interest and study $(13,15)$, many cell cycle regulators in trypanosomatids remain to be identified and much remains to be learned about cell cycle control and progression in these parasites. DNA staining followed by flow cytometry is a widely used approach for quantifying cellular DNA content and analysing cell cycle distribution across otherwise asynchronous populations. Here, we combined genome scale loss-of-function genetic screening with flow cytometry in bloodstream form African trypanosomes and identify hundreds of genes required for progression through specific stages of the cell cycle.

Functional annotation of the trypanosomatid genomes will continue to benefit from novel high-throughput functional analyses, and RNAi-mediated knockdown has proven to be a powerful approach for T. brucei. RIT-seq profiling provides data for almost every gene and, using this approach, we previously described genome-scale loss-of-fitness data (23). Amongst 3117 knockdowns that scored a significant loss-of-fitness in bloodstream-form cells in that screen ( $42 \%$ of all genes analysed) were genes encoding all 18 intraflagellar transport complex subunits $\left(\chi^{2} p=1^{-6}\right), 12$ of 13 dynein heavy-chains $\left(\chi^{2} p=4^{-4}\right)$, all 8 TCP-1 chaperone components $\left(\chi^{2} p=1^{-3}\right), 27$ of 30 nucleoporins $\left(\chi^{2} p=2^{-7}\right)$, all eleven glycolytic enzymes $\left(\chi^{2} p=2^{-4}\right)$ and 30 of 31 proteasome subunits $\left(\chi^{2} p=2^{-9}\right)$. This set also included 18 of 19 kinetochore proteins $\left(\chi^{2} p=6^{-6}\right)$, only later identified as components of this essential complex (17). With this study, we now link many of these genes and many more to specific cell cycle defects following RNAi knockdown. A large number of flagellar protein knockdowns, in particular, yield cells with excess DNA, suggesting that DNA replication typically continues following failure to complete cytokinesis. We identified a number of pathways and protein complexes that impact cell cycle progression, such as glycolysis $\left(\mathrm{G}_{1} / \mathrm{S}\right.$ transition) and the proteasome (likely $\mathrm{G}_{2} / \mathrm{M}$ transition). We also identify many mRNA binding proteins and protein kinases implicated in control of cell cycle progression. Notably, we link multiple known potential and promising drug targets to cell cycle progression defects, such as glycolytic enzymes (86), the proteasome $(87)$, kinetochore kinases $(74,88)$ and other kinases (89).

Prior cell cycle studies have often focused on trypanosome orthologues of known regulators from other eukaryotes. Since genome-scale profiling is unbiased, it presents the opportunity to uncover divergent as well as novel factors and regulators that impact cell cycle progression. Accordingly, we link many previously uncharacterised and hypothetical proteins of unknown function to specific cell cycle progression defects. Thus, we uncover mechanisms with an ancient origin in a common eukaryotic ancestor and others likely 
523 reflecting trypanosomatid-specific biology. We also compared our functional data with cell

524 cycle regulated transcriptome and (phospho)proteome datasets.

The digital dataset provided in Supplementary File 1 facilitates further interrogation and further analysis of the genome-scale cell cycle RIT-seq data. We have also made the data available via an interactive, open access, online data visualization tool, which allows searching and browsing of the data (see Figure 2-figure supplement 2). Comparison with existing and new datasets, including with high-throughput subcellular localisation data (90) www.tryptag.org, should also facilitate future studies. Since high-throughput genetic screens typically yield a proportion of false positive 'hits', we do urge some caution, however, in particular where outputs are predominantly generated by a single RIT-seq fragment. On the other hand, there are knockdowns in the current dataset that show specific cell cycle phase enrichment yet fail to register a sufficient enrichment to surpass the thresholds applied above. Considering both of these points, we hope that the digital dataset and the online database will serve as valuable resources. Since other important trypanosomatid parasites, including Trypanosoma cruzi and Leishmania, share a high degree of conservation and synteny with $T$. brucei (91), the current datasets can also assist and inform studies on other trypanosomatids.

In summary, we report RNAi induced cell cycle defects at a genomic scale and identify the $T$. brucei genes that underlie these defects. The outputs confirm known roles in cell cycle progression and provide functional annotation for many additional genes, including many with no prior functional assignment and many that are trypanosomatid-specific. As

544 such, the data not only improve our understanding of cell cycle progression in these 545 important and divergent pathogens but should also accelerate further discovery. Taken

546 together, our findings further facilitate genome annotation, drug-target prioritisation and provide comprehensive genetic evidence for the protein complexes, pathways and regulatory factors that coordinate progression through the trypanosome cell cycle. 


\section{T. brucei RNAi library growth and manipulation}

552 The bloodstream form T. brucei RNAi library (22) was thawed in HMI-11 containing $1 \mu$ g.ml-1 553 of blasticidin and $0.2 \mu \mathrm{g} \cdot \mathrm{ml}^{-1}$ of phleomycin and incubated at $37^{\circ} \mathrm{C}$ in $5 \% \mathrm{CO}_{2}$. After

554 approximately $48 \mathrm{~h}$, six flasks, each containing $2 \times 10^{7}$ cells in $150 \mathrm{ml}$ of HMI-11 as above, were prepared; $1 \mu \mathrm{g} \cdot \mathrm{ml}^{-1}$ of tetracycline was added to five of them, while one served as the non-induced control. The cells were grown under these conditions for $24 \mathrm{~h}$ and then harvested by centrifugation for $10 \mathrm{~min}$ at $1000 \mathrm{~g}$. Cells from each flask were then resuspended in $25 \mathrm{ml}$ of $1 \mathrm{x}$ PBS (pH 7.0) supplemented with $5 \mathrm{mM}$ EDTA and $1 \%$ FBS ("supplemented PBS"), centrifuged again for $10 \mathrm{~min}$ at $1000 \mathrm{~g}$, and then re-suspended in 0.5 $\mathrm{ml}$ of supplemented PBS. To each cell suspension, $9.5 \mathrm{ml}$ of $1 \%$ formaldehyde in supplemented PBS was added dropwise, with regular vortexing. The cells were incubated for a further $10 \mathrm{~min}$ at room temperature and then washed twice in $10 \mathrm{ml}$ of supplemented PBS using centrifugation as above. The cells were finally re-suspended at $2.5 \times 10^{7}$ per $\mathrm{ml}$ in supplemented PBS and were subsequently stored at $4^{\circ} \mathrm{C}$, in the dark.

\section{Flow cytometry}

567

Fixed cells, $3 \times 10^{8}$ Tet-induced and $10^{7}$ uninduced, were centrifuged for $10 \mathrm{~min}$ at $1000 \mathrm{~g}$, and re-suspended in $10 \mathrm{ml}$ of supplemented PBS containing $0.01 \%$ Triton X-100 (Sigma Aldrich). The cells were incubated for $30 \mathrm{~min}$ at room temperature, centrifuged for $10 \mathrm{~min}$ at $700 \mathrm{~g}$ and washed once in $10 \mathrm{ml}$ of supplemented PBS. The cells were then re-suspended in $4 \mathrm{ml}$ of supplemented PBS containing $10 \mu \mathrm{g} \cdot \mathrm{ml}^{-1}$ of propidium iodide (Sigma Aldrich) and $100 \mu \mathrm{g} \cdot \mathrm{ml}^{-1}$ of RNaseA (Sigma Aldrich), and incubated for $45 \mathrm{~min}$ at $37^{\circ} \mathrm{C}$, in the dark; cells were subsequently kept on ice and in the dark. Immediately prior to sorting, the Tet-induced cells were filtered (Filcon Cup-type filter, $50 \mu \mathrm{m}$ mesh, BD ${ }^{\mathrm{TM}}$ Medimachine) into $5 \mathrm{ml}$ polystyrene round-bottom tubes (BD Falcon). Cells were sorted using the BD Influx ${ }^{\mathrm{TM}}$ (Becton Dickinson) cell sorter, with BD FACSort ${ }^{\mathrm{TM}}$ software, at the Flow Cytometry and Cell Sorting Facility in the School of Life Sciences, University of Dundee. The cells were sorted into pools of $<2 \mathrm{C}\left(\sim 8 \times 10^{5}\right.$ cells), $2 \mathrm{C}$ ( $\mathrm{G}_{1}, 1 \times 10^{7}$ cells), $2-4 \mathrm{C}\left(\mathrm{S}, 1 \times 10^{7}\right.$ cells), $4 \mathrm{C}\left(\mathrm{G}_{2} \mathrm{M}, 1 \times\right.$ $10^{7}$ cells $)$ and $>4 \mathrm{C}\left(\sim 5 \times 10^{5}\right.$ cells $)$ based on their DNA content, and collected into $50 \mathrm{ml}$ Falcon tubes (BD Falcon); sorting time was approx. $4 \mathrm{~h}$. The 2C, 2-4C and $4 \mathrm{C}$ sorted samples were then run on a FACS LSR Fortessa flow cytometry analyser for a post-sorting quality check. FlowJo v10 was used for data analysis and visualisation. 
The five pools of Tet-induced, sorted cells as well as uninduced or induced, but unsorted

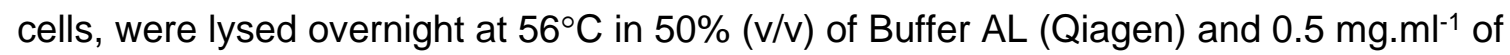
Proteinase K (Qiagen), to reverse formaldehyde crosslinking. Genomic DNA was then extracted using the DNeasy Blood and Tissue DNA extraction kit (Qiagen), according to the manufacturer's instructions, with the exception that each sample was eluted in $50 \mu \mathrm{l}$ of Buffer AE. The whole sample (range = 140-840 ng) was used for PCR, in a $100 \mu \mathrm{l}$ reaction, using OneTaq (NEB), and the Lib3F (CCTCGAGGGCCAGTGAG) and Lib3R (ATCAAGCTTGGCCTGTGAG) primers and with the following programme: $94^{\circ} \mathrm{C}$ for $4 \mathrm{~min}$, followed by 27 cycles of $94^{\circ} \mathrm{C}$ for $30 \mathrm{sec}, 55^{\circ} \mathrm{C}$ for $30 \mathrm{sec}$ and $68^{\circ} \mathrm{C}$ for $2 \mathrm{~min}$ and $10 \mathrm{sec}$, and a final extension of $68^{\circ} \mathrm{C}$ for $5 \mathrm{~min}$. The PCR products were then purified using the Qiaquick PCR extraction kit (Qiagen), as per the manufacturer's instructions, and eluted in $30 \mu$ of nuclease-free water (Ambion); two columns per sample.

\section{RIT-seq library preparation and sequencing}

600 Purified PCR products were used for library preparation and sequencing at the Tayside

601 Centre for Genomic Analysis at the University of Dundee. The PCR products were fragmented using a Covaris M220 sonicator (20\% duty factor, $75 \mathrm{~W}$ peak/displayed power, 60 seconds duration $-3 \times 20 \mathrm{sec}$ with intermittent spin down step, $18-20^{\circ} \mathrm{C}$ temperature; resulting in 250-300 bp enriched fragments), and the libraries were prepared using the Truseq Nano DNA Library Prep kit (Illumina). The samples were multiplexed, and sequenced on an Illumina NextSeq 500 platform, on a 150 cycle Output Cartridge v2, paired-end. Each library was run on 4 sequencing lanes. Base call, index deconvolution, trimming and QC were performed in BaseSpace using bcl2fastq2 Conversion Software v2.17.

\section{RIT-seq data mapping and analysis}

The sequencing data analysis pipeline was adapted from (22). The FASTQ files with forward and reverse paired end reads (4 technical replicates for each samples) were concatenated and aligned to the reference genome v46 of T. brucei clone TREU927 downloaded from TriTrypDB (28) using Bowtie2 (92), with the 'very-sensitive-local' pre-set alignment option. The alignments were converted to BAM format, reference sorted and indexed with SAMtools (93). The quality of alignments was evaluated with Qualimap 2 (94) using the bamqc and rnaseq options. The Qualimap 2 output files were aggregated with MultiQC (95) and inspected. The alignments were deduplicated with the Picard tools package using the MarkDuplicates function (http://broadinstitute.github.io/picard/); to 
621 with properly paired reads were extracted with SAMtool view using the $-f 2$ option and

622 parsed with a custom python script to extract the paired reads containing the barcode

623 sequence (GTGAGGCCTCGCGA) in forward or reverse complement orientation. The

624 genome coverage of the aligned reads was extracted from the bam files using bedtools (96)

625 with the -bg option to output bedGraph files. The bedGraph files were visualized with the

626 svist4get python package (97). Read counts for protein coding sequences and associated

627 untranslated regions (where annotated) were determined from the bam files using

628 featureCounts (98) and normalized to Transcripts Per Kilobase Million (TPM). Dimensionality

629 reduction of the $\mathrm{G}_{1}, \mathrm{~S}$ and $\mathrm{G}_{2} \mathrm{M}$ TPM values was performed with the radviz algoritm

630 implemented in the pandas python package (99). The bash script containing the analysis

631 pipline, a conda environment specification file for its execution, the python script to extract

632 barcoded reads and a basic usage example are available at GitHub

633 (https://github.com/mtinti/ritseq_cellcycle). Data were subsequently analysed using a GO-

634 slim set and Gene Ontology tools available via tritrypdb.org and visualised using tools

635 available at huygens.science.uva.nl/PlotsOfData. 


\section{Acknowledgements}

637 We thank R. Clark, A. Rennie and M. Lee of the Flow Cytometry and Cell Sorting Facility,

638 which is supported by the Wellcome Trust (097418/Z/11/Z). We also thank L. Glover for

639 advice on RNAi library manipulation, S. Hutchinson for advice on RIT-seq data analysis and

640 J. Faria for fruitful discussions.

641

642 Funding: The work was funded by a Wellcome Trust Investigator Award to D.H.

643 [100320/Z/12/Z]. The funders had no role in study design, data collection and interpretation,

644 or the decision to submit the work for publication.

645

646 Author contributions

647 Catarina A. Marques, Conceptualization, Formal analysis, Investigation, Methodology,

648 Writing — review and editing; Michele Tinti, Data curation, visualisation and analysis,

649 Writing — review and editing; Andrew Cassidy, Investigation, Methodology; David Horn,

650 Conceptualization, Formal analysis, Supervision, Funding acquisition, Project administration,

651 Writing—original draft, Writing—review and editing.

652

\section{Author ORCIDs}

654 David Horn http://orcid.org/0000-0001-5173-9284

655 Catarina A. Marques https://orcid.org/0000-0003-1324-5448

656 Michele Tinti https://orcid.org/0000-0002-0051-017X

657

658 Competing interests: The authors declare that they have no competing interests.

659

660 Data and materials availability: High-throughput sequencing data generated for

661 this study have been deposited in the Short Read Archive (SRA) at

662 https://www.ncbi.nlm.nih.gov/sra/PRJNA641153 under primary accession number

663 PRJNA641153. 


\section{References}

665 1. Harashima, H., Dissmeyer, N. and Schnittger, A. (2013) Cell cycle control across the eukaryotic kingdom. Trends Cell Biol, 23, 345-356.

667

2. Pollard, T.D. and O'Shaughnessy, B. (2019) Molecular mechanism of mytokinesis.

668

669 Annu Rev Biochem, 88, 661-689.

3. Poon, R.Y. (2016) Cell cycle control: A system of interlinking oscillators. Methods Mol Biol, 1342, 3-19.

670

671

672

673

4. Visconti, R., Della Monica, R. and Grieco, D. (2016) Cell cycle checkpoint in cancer: a therapeutically targetable double-edged sword. J Exp Clin Cancer Res, 35, 153.

5. Buscher, P., Cecchi, G., Jamonneau, V. and Priotto, G. (2017) Human African trypanosomiasis. Lancet, 390, 2397-2409.

674

675

6. Matthews, K.R. (2015) 25 years of African trypanosome research: From description

676

677 to molecular dissection and new drug discovery. Mol Biochem Parasitol, 200, 30-40.

7. Broadhead, R., Dawe, H.R., Farr, H., Griffiths, S., Hart, S.R., Portman, N., Shaw, M.K., Ginger, M.L., Gaskell, S.J., McKean, P.G. et al. (2006) Flagellar motility is required for the viability of the bloodstream trypanosome. Nature, 440, 224-227.

8. Baron, D.M., Kabututu, Z.P. and Hill, K.L. (2007) Stuck in reverse: loss of LC1 in Trypanosoma brucei disrupts outer dynein arms and leads to reverse flagellar beat and backward movement. J Cell Sci, 120, 1513-1520.

683

9. Bonnefoy, S., Watson, C.M., Kernohan, K.D., Lemos, M., Hutchinson, S., Poulter, J.A., Crinnion, L.A., Berry, I., Simmonds, J., Vasudevan, P. et al. (2018) Biallelic mutations in LRRC56, encoding a protein associated with intraflagellar transport, cause mucociliary clearance and laterality defects. Am J Hum Genet, 103, 727-739.

687

688

10. Coutton, C., Vargas, A.S., Amiri-Yekta, A., Kherraf, Z.E., Ben Mustapha, S.F., Le Tanno, P., Wambergue-Legrand, C., Karaouzene, T., Martinez, G., Crouzy, S. et al. (2018) Mutations in CFAP43 and CFAP44 cause male infertility and flagellum defects in Trypanosoma and human. Nat Commun, 9, 686.

11. Vincensini, L., Blisnick, T. and Bastin, P. (2011) [The importance of model organisms to study cilia and flagella biology]. Biol Aujourdhui, 205, 5-28.

12. Allmann, S. and Bringaud, F. (2017) Glycosomes: A comprehensive view of their metabolic roles in T. brucei. Int J Biochem Cell Biol, 85, 85-90.

13. Wheeler, R.J., Gull, K. and Sunter, J.D. (2019) Coordination of the cell cycle in trypanosomes. Annu Rev Microbiol, 73, 133-154.

14. Clayton, C. (2019) Regulation of gene expression in trypanosomatids: living with polycistronic transcription. Open Biol, 9, 190072.

15. Zhou, Q., Hu, H. and Li, Z. (2014) New insights into the molecular mechanisms of mitosis and cytokinesis in trypanosomes. Int Rev Cell Mol Biol, 308, 127-166.

16. Ploubidou, A., Robinson, D.R., Docherty, R.C., Ogbadoyi, E.O. and Gull, K. (1999) Evidence for novel cell cycle checkpoints in trypanosomes: kinetoplast segregation and cytokinesis in the absence of mitosis. J Cell Sci, 112 ( Pt 24), 4641-4650.

17. Akiyoshi, B. and Gull, K. (2014) Discovery of unconventional kinetochores in kinetoplastids. Cell, 156, 1247-1258.

18. Marques, C.A. and McCulloch, R. (2018) Conservation and variation in strategies for DNA replication of kinetoplastid nuclear genomes. Curr Genomics, 19, 98-109.

19. Archer, S.K., Inchaustegui, D., Queiroz, R. and Clayton, C. (2011) The cell cycle regulated transcriptome of Trypanosoma brucei. PLoS One, 6, e18425.

20. Crozier, T.W.M., Tinti, M., Wheeler, R.J., Ly, T., Ferguson, M.A.J. and Lamond, A.I. (2018) Proteomic analysis of the cell cycle of procylic form Trypanosoma brucei. Mol Cell Proteomics, 17, 1184-1195. 
21. Benz, C. and Urbaniak, M.D. (2019) Organising the cell cycle in the absence of transcriptional control: Dynamic phosphorylation co-ordinates the Trypanosoma brucei cell cycle post-transcriptionally. PLoS Pathog, 15, e1008129.

22. Glover, L., Alsford, S., Baker, N., Turner, D.J., Sanchez-Flores, A., Hutchinson, S., Hertz-Fowler, C., Berriman, M. and Horn, D. (2015) Genome-scale RNAi screens for high-throughput phenotyping in bloodstream-form African trypanosomes. Nat Protoc, 10, 106-133.

23. Alsford, S., Turner, D.J., Obado, S.O., Sanchez-Flores, A., Glover, L., Berriman, M., Hertz-Fowler, C. and Horn, D. (2011) High-throughput phenotyping using parallel sequencing of RNA interference targets in the African trypanosome. Genome Res, 21, 915-924.

24. Ngo, H., Tschudi, C., Gull, K. and Ullu, E. (1998) Double-stranded RNA induces mRNA degradation in Trypanosoma brucei. Proc Natl Acad Sci U S A, 95, 1468714692.

25. Hammarton, T.C., Clark, J., Douglas, F., Boshart, M. and Mottram, J.C. (2003) Stagespecific differences in cell cycle control in Trypanosoma brucei revealed by RNA interference of a mitotic cyclin. J Biol Chem, 278, 22877-22886.

26. Li, Z., Umeyama, T. and Wang, C.C. (2008) The chromosomal passenger complex and a mitotic kinesin interact with the tousled-like kinase in trypanosomes to regulate mitosis and cytokinesis. PLoS One, 3, e3814.

27. Kolev, N.G., Tschudi, C. and Ullu, E. (2011) RNA interference in protozoan parasites: achievements and challenges. Eukaryot Cell, 10, 1156-1163.

28. Aslett, M., Aurrecoechea, C., Berriman, M., Brestelli, J., Brunk, B.P., Carrington, M., Depledge, D.P., Fischer, S., Gajria, B., Gao, X. et al. (2010) TriTrypDB: a functional genomic resource for the Trypanosomatidae. Nucleic Acids Res, 38, D457-462.

29. Springer, A.L., Bruhn, D.F., Kinzel, K.W., Rosenthal, N.F., Zukas, R. and Klingbeil, M.M. (2011) Silencing of a putative inner arm dynein heavy chain results in flagellar immotility in Trypanosoma brucei. Mol Biochem Parasitol, 175, 68-75.

30. Gassen, A., Brechtefeld, D., Schandry, N., Arteaga-Salas, J.M., Israel, L., Imhof, A. and Janzen, C.J. (2012) DOT1A-dependent H3K76 methylation is required for replication regulation in Trypanosoma brucei. Nucleic Acids Res, 40, 10302-10311.

31. Choe, K.N. and Moldovan, G.L. (2017) Forging ahead through darkness: PCNA, still the principal conductor at the replication fork. Mol Cell, 65, 380-392.

32. Valenciano, A.L., Ramsey, A.C. and Mackey, Z.B. (2015) Deviating the level of proliferating cell nuclear antigen in Trypanosoma brucei elicits distinct mechanisms for inhibiting proliferation and cell cycle progression. Cell Cycle, 14, 674-688.

33. Rudd, S.G., Glover, L., Jozwiakowski, S.K., Horn, D. and Doherty, A.J. (2013) PPL2 translesion polymerase is essential for the completion of chromosomal DNA replication in the African trypanosome. Mol Cell, 52, 554-565.

34. Ralston, K.S., Lerner, A.G., Diener, D.R. and Hill, K.L. (2006) Flagellar motility contributes to cytokinesis in Trypanosoma brucei and is modulated by an evolutionarily conserved dynein regulatory system. Eukaryot Cell, 5, 696-711.

35. Boehm, C.M., Obado, S., Gadelha, C., Kaupisch, A., Manna, P.T., Gould, G.W., Munson, M., Chait, B.T., Rout, M.P. and Field, M.C. (2017) The trypanosome exocyst: A conserved structure revealing a new role in endocytosis. PLoS Pathog, 13, e1006063.

36. Wang, D.Y., Kamuda, K., Montoya, G. and Mesa, P. (2020) The TRiC/CCT chaperonin and its role in uncontrolled proliferation. Adv Exp Med Biol, 1243, 21-40. 
37. Zhang, X., Hu, H., Lun, Z.R. and Li, Z. (2019) Functional analyses of an axonemal inner-arm dynein complex in the bloodstream form of Trypanosoma brucei uncover its essential role in cytokinesis initiation. Mol Microbiol, 112, 1718-1730.

38. Sunter, J.D., Varga, V., Dean, S. and Gull, K. (2015) A dynamic coordination of flagellum and cytoplasmic cytoskeleton assembly specifies cell morphogenesis in trypanosomes. J Cell Sci, 128, 1580-1594.

39. Hu, H., Majneri, P., Li, D., Kurasawa, Y., An, T., Dong, G. and Li, Z. (2017) Functional analyses of the CIF1-CIF2 complex in trypanosomes identify the structural motifs required for cytokinesis. J Cell Sci, 130, 4108-4119.

40. Li, Z., Lee, J.H., Chu, F., Burlingame, A.L., Gunzl, A. and Wang, C.C. (2008) Identification of a novel chromosomal passenger complex and its unique localization during cytokinesis in Trypanosoma brucei. PLoS One, 3, e2354.

41. Li, Z. and Wang, C.C. (2006) Changing roles of aurora-B kinase in two life cycle stages of Trypanosoma brucei. Eukaryot Cell, 5, 1026-1035.

42. Jones, N.G., Thomas, E.B., Brown, E., Dickens, N.J., Hammarton, T.C. and Mottram, J.C. (2014) Regulators of Trypanosoma brucei cell cycle progression and differentiation identified using a kinome-wide RNAi screen. PLoS Pathog, 10, e1003886.

43. Hu, H., Liu, Y., Zhou, Q., Siegel, S. and Li, Z. (2015) The centriole cartwheel protein SAS-6 in Trypanosoma brucei is required for probasal body biogenesis and flagellum assembly. Eukaryot Cell, 14, 898-907.

44. Zhang, X., An, T., Pham, K.T.M., Lun, Z.R. and Li, Z. (2019) Functional analyses of cytokinesis regulators in bloodstream stage Trypanosoma brucei parasites identify functions and regulations specific to the life cycle stage. mSphere, 4.

45. Dean, S., Moreira-Leite, F., Varga, V. and Gull, K. (2016) Cilium transition zone proteome reveals compartmentalization and differential dynamics of ciliopathy complexes. Proc Natl Acad Sci U S A, 113, E5135-5143.

46. Dang, H.Q., Zhou, Q., Rowlett, V.W., Hu, H., Lee, K.J., Margolin, W. and Li, Z. (2017) Proximity interactions among basal body components in Trypanosoma brucei identify novel regulators of basal body biogenesis and inheritance. mBio, $\mathbf{8}$.

47. An, T. and Li, Z. (2018) An orphan kinesin controls trypanosome morphology transitions by targeting FLAM3 to the flagellum. PLoS Pathog, 14, e1007101.

48. Hu, L., Hu, H. and Li, Z. (2012) A kinetoplastid-specific kinesin is required for cytokinesis and for maintenance of cell morphology in Trypanosoma brucei. Mol Microbiol, 83, 565-578.

49. DeGrasse, J.A., DuBois, K.N., Devos, D., Siegel, T.N., Sali, A., Field, M.C., Rout, M.P. and Chait, B.T. (2009) Evidence for a shared nuclear pore complex architecture that is conserved from the last common eukaryotic ancestor. Mol Cell Proteomics, 8 , 2119-2130.

50. Benz, C., Clucas, C., Mottram, J.C. and Hammarton, T.C. (2012) Cytokinesis in bloodstream stage Trypanosoma brucei requires a family of katanins and spastin. PLoS One, 7, e30367.

51. Ralston, K.S. and Hill, K.L. (2006) Trypanin, a component of the flagellar dynein regulatory complex, is essential in bloodstream form African trypanosomes. PLoS Pathog, 2, e101.

52. May, S.F., Peacock, L., Almeida Costa, C.I., Gibson, W.C., Tetley, L., Robinson, D.R. and Hammarton, T.C. (2012) The Trypanosoma brucei AIR9-like protein is cytoskeleton-associated and is required for nucleus positioning and accurate cleavage furrow placement. Mol Microbiol, 84, 77-92. 
53. Portman, N. and Gull, K. (2014) Identification of paralogous life-cycle stage specific cytoskeletal proteins in the parasite Trypanosoma brucei. PLoS One, 9, e106777.

54. Dostalova, A., Kaser, S., Cristodero, M. and Schimanski, B. (2013) The nuclear mRNA export receptor Mex67-Mtr2 of Trypanosoma brucei contains a unique and essential zinc finger motif. Mol Microbiol, 88, 728-739.

55. Marques, C.A., Tiengwe, C., Lemgruber, L., Damasceno, J.D., Scott, A., Paape, D., Marcello, L. and McCulloch, R. (2016) Diverged composition and regulation of the Trypanosoma brucei origin recognition complex that mediates DNA replication initiation. Nucleic Acids Res, 44, 4763-4784.

56. Gluenz, E., Sharma, R., Carrington, M. and Gull, K. (2008) Functional characterization of cohesin subunit SCC1 in Trypanosoma brucei and dissection of mutant phenotypes in two life cycle stages. Mol Microbiol, 69, 666-680.

57. Dang, H.Q. and Li, Z. (2011) The Cdc45.Mcm2-7.GINS protein complex in trypanosomes regulates DNA replication and interacts with two Orc1-like proteins in the origin recognition complex. J Biol Chem, 286, 32424-32435.

58. Shyh-Chang, N., Locasale, J.W., Lyssiotis, C.A., Zheng, Y., Teo, R.Y., Ratanasirintrawoot, S., Zhang, J., Onder, T., Unternaehrer, J.J., Zhu, H. et al. (2013) Influence of threonine metabolism on S-adenosylmethionine and histone methylation. Science, 339, 222-226.

59. Peacock, L., Bailey, M., Carrington, M. and Gibson, W. (2014) Meiosis and haploid gametes in the pathogen Trypanosoma brucei. Curr Biol, 24, 181-186.

60. Ammerman, M.L., Downey, K.M., Hashimi, H., Fisk, J.C., Tomasello, D.L., Faktorova, D., Kafkova, L., King, T., Lukes, J. and Read, L.K. (2012) Architecture of the trypanosome RNA editing accessory complex, MRB1. Nucleic Acids Res, 40, 5637-5650.

61. Zikova, A., Schnaufer, A., Dalley, R.A., Panigrahi, A.K. and Stuart, K.D. (2009) The F0F1-ATP synthase complex contains novel subunits and is essential for procyclic Trypanosoma brucei. PLoS Pathog, 5, e1000436.

62. Tudzarova, S., Colombo, S.L., Stoeber, K., Carcamo, S., Williams, G.H. and Moncada, S. (2011) Two ubiquitin ligases, APC/C-Cdh1 and SKP1-CUL1-F (SCF)beta-TrCP, sequentially regulate glycolysis during the cell cycle. Proc Natl Acad Sci U S A, 108, 5278-5283.

63. Hu, J.W., Sun, P., Zhang, D.X., Xiong, W.J. and Mi, J. (2014) Hexokinase 2 regulates G1/S checkpoint through CDK2 in cancer-associated fibroblasts. Cell Signal, 26, 2210-2216.

64. Morgan, G.A., Hamilton, E.A. and Black, S.J. (1996) The requirements for G1 checkpoint progression of Trypanosoma brucei S 427 clone 1. Mol Biochem Parasitol, 78, 195-207.

65. Silvester, E., McWilliam, K.R. and Matthews, K.R. (2017) The cytological events and molecular control of life cycle development of Trypanosoma brucei in the mammalian bloodstream. Pathogens, 6.

66. Qiu, Y., Milanes, J.E., Jones, J.A., Noorai, R.E., Shankar, V. and Morris, J.C. (2018) Glucose signaling is important for nutrient adaptation during differentiation of pleomorphic African trypanosomes. mSphere, 3.

67. Naguleswaran, A., Doiron, N. and Roditi, I. (2018) RNA-Seq analysis validates the use of culture-derived Trypanosoma brucei and provides new markers for mammalian and insect life-cycle stages. BMC Genomics, 19, 227.

68. Kalucka, J., Missiaen, R., Georgiadou, M., Schoors, S., Lange, C., De Bock, K., Dewerchin, M. and Carmeliet, P. (2015) Metabolic control of the cell cycle. Cell Cycle, 14, 3379-3388. 
69. Burgers, P.M.J. and Kunkel, T.A. (2017) Eukaryotic DNA replication fork. Annu Rev Biochem, 86, 417-438.

70. Hayashi, H. and Akiyoshi, B. (2018) Degradation of cyclin B is critical for nuclear division in Trypanosoma brucei. Biol Open, 7.

71. Benz, C. and Clayton, C.E. (2007) The F-box protein CFB2 is required for cytokinesis of bloodstream-form Trypanosoma brucei. Mol Biochem Parasitol, 156, 217-224.

72. Van Hellemond, J.J. and Mottram, J.C. (2000) The CYC3 gene of Trypanosoma brucei encodes a cyclin with a short half-life. Mol Biochem Parasitol, 111, 275-282.

73. Ishii, M. and Akiyoshi, B. (2020) Characterization of unconventional kinetochore kinases KKT10 and KKT19 in Trypanosoma brucei. J Cell Sci, 133.

74. Saldivia, M., Fang, E., Ma, X., Myburgh, E., Carnielli, J.B.T., Bower-Lepts, C., Brown, E., Ritchie, R., Lakshminarayana, S.B., Chen, Y.-L. et al. (2020) Targeting the trypanosome kinetochore with CLK1 protein kinase inhibitors. Nature Microbiology.

75. Hinnebusch, A.G. (2006) eIF3: a versatile scaffold for translation initiation complexes. Trends Biochem Sci, 31, 553-562.

76. Mugo, E. and Clayton, C. (2017) Expression of the RNA-binding protein RBP10 promotes the bloodstream-form differentiation state in Trypanosoma brucei. PLoS Pathog, 13, e1006560.

77. Hammarton, T.C., Kramer, S., Tetley, L., Boshart, M. and Mottram, J.C. (2007) Trypanosoma brucei Polo-like kinase is essential for basal body duplication, kDNA segregation and cytokinesis. Mol Microbiol, 65, 1229-1248.

78. Kumar, P. and Wang, C.C. (2006) Dissociation of cytokinesis initiation from mitotic control in a eukaryote. Eukaryot Cell, 5, 92-102.

79. McAllaster, M.R., Ikeda, K.N., Lozano-Nunez, A., Anrather, D., Unterwurzacher, V., Gossenreiter, T., Perry, J.A., Crickley, R., Mercadante, C.J., Vaughan, S. et al. (2015) Proteomic identification of novel cytoskeletal proteins associated with TbPLK, an essential regulator of cell morphogenesis in Trypanosoma brucei. Mol Biol Cell, 26, 3013-3029.

80. Tu, X. and Wang, C.C. (2004) The involvement of two cdc2-related kinases (CRKs) in Trypanosoma brucei cell cycle regulation and the distinctive stage-specific phenotypes caused by CRK3 depletion. J Biol Chem, 279, 20519-20528.

81. Menzies, S.K., Tulloch, L.B., Florence, G.J. and Smith, T.K. (2018) The trypanosome alternative oxidase: a potential drug target? Parasitology, 145, 175-183.

82. Li, Z. and Wang, C.C. (2003) A PHO80-like cyclin and a B-type cyclin control the cell cycle of the procyclic form of Trypanosoma brucei. J Biol Chem, 278, 2065220658.

83. Wei, Y., Hu, H., Lun, Z.R. and Li, Z. (2014) Centrin3 in trypanosomes maintains the stability of a flagellar inner-arm dynein for cell motility. Nat Commun, 5, 4060.

84. Denninger, V. and Rudenko, G. (2014) FACT plays a major role in histone dynamics affecting VSG expression site control in Trypanosoma brucei. Mol Microbiol, 94, 945-962.

85. Prendergast, L., Muller, S., Liu, Y., Huang, H., Dingli, F., Loew, D., Vassias, I., Patel, D.J., Sullivan, K.F. and Almouzni, G. (2016) The CENP-T/-W complex is a binding partner of the histone chaperone FACT. Genes Dev, 30, 1313-1326.

86. Haanstra, J.R., Gerding, A., Dolga, A.M., Sorgdrager, F.J.H., Buist-Homan, M., du Toit, F., Faber, K.N., Holzhutter, H.G., Szoor, B., Matthews, K.R. et al. (2017) Targeting pathogen metabolism without collateral damage to the host. Sci Rep, 7, 40406. 
942

943

944

945

946

87. Khare, S., Nagle, A.S., Biggart, A., Lai, Y.H., Liang, F., Davis, L.C., Barnes, S.W., Mathison, C.J., Myburgh, E., Gao, M.Y. et al. (2016) Proteasome inhibition for treatment of leishmaniasis, Chagas disease and sleeping sickness. Nature, 537, 229233.

88. Torrie, L.S., Zuccotto, F., Robinson, D.A., Gray, D.W., Gilbert, I.H. and De Rycker, M. (2019) Identification of inhibitors of an unconventional Trypanosoma brucei kinetochore kinase. PLoS One, 14, e0217828.

89. Urbaniak, M.D., Mathieson, T., Bantscheff, M., Eberhard, D., Grimaldi, R., MirandaSaavedra, D., Wyatt, P., Ferguson, M.A., Frearson, J. and Drewes, G. (2012) Chemical proteomic analysis reveals the drugability of the kinome of Trypanosoma brucei. ACS Chem Biol, 7, 1858-1865.

90. Halliday, C., Billington, K., Wang, Z., Madden, R., Dean, S., Sunter, J.D. and Wheeler, R.J. (2019) Cellular landmarks of Trypanosoma brucei and Leishmania mexicana. Mol Biochem Parasitol, 230, 24-36.

91. El-Sayed, N.M., Myler, P.J., Blandin, G., Berriman, M., Crabtree, J., Aggarwal, G., Caler, E., Renauld, H., Worthey, E.A., Hertz-Fowler, C. et al. (2005) Comparative genomics of trypanosomatid parasitic protozoa. Science, 309, 404-409.

92. Langmead, B. and Salzberg, S.L. (2012) Fast gapped-read alignment with Bowtie 2. Nat Methods, 9, 357-359.

93. Li, H., Handsaker, B., Wysoker, A., Fennell, T., Ruan, J., Homer, N., Marth, G., Abecasis, G., Durbin, R. and Genome Project Data Processing, S. (2009) The Sequence Alignment/Map format and SAMtools. Bioinformatics, 25, 2078-2079.

94. Okonechnikov, K., Conesa, A. and Garcia-Alcalde, F. (2016) Qualimap 2: advanced multi-sample quality control for high-throughput sequencing data. Bioinformatics, 32, 292-294.

95. Ewels, P., Magnusson, M., Lundin, S. and Kaller, M. (2016) MultiQC: summarize analysis results for multiple tools and samples in a single report. Bioinformatics, 32, 3047-3048.

96. Quinlan, A.R. and Hall, I.M. (2010) BEDTools: a flexible suite of utilities for comparing genomic features. Bioinformatics, 26, 841-842.

97. Egorov, A.A., Sakharova, E.A., Anisimova, A.S., Dmitriev, S.E., Gladyshev, V.N. and Kulakovskiy, I.V. (2019) svist4get: a simple visualization tool for genomic tracks from sequencing experiments. BMC Bioinformatics, 20, 113.

98. Liao, Y., Smyth, G.K. and Shi, W. (2014) featureCounts: an efficient general purpose program for assigning sequence reads to genomic features. Bioinformatics, 30, 923930.

99. Virtanen, P., Gommers, R., Oliphant, T.E., Haberland, M., Reddy, T., Cournapeau, D., Burovski, E., Peterson, P., Weckesser, W., Bright, J. et al. (2020) SciPy 1.0: fundamental algorithms for scientific computing in Python. Nat Methods, 17, 261272. 


\section{Figure Legends}

953 Figure 1. A genome-wide conditional knockdown screen for cell cycle progression defects.

954 (A) Schematic representation of the bloodstream form T. brucei cell cycle, also showing

955 aberrant sub-2C and $>4$ C phenotypes. (B) The schematic illustrates the RIT-seq screen;

956 massive parallel induction of RNAi followed by flow cytometry and RIT-seq,

957 allowing for reconstruction of cell cycle profiles, using mapped reads from each knockdown.

958 Each read-mapping profile encompasses the gene of interest and associated untranslated

959 regions present in the cognate mRNA. The library data represents the uninduced and

960 unsorted population. GenelDs, Tb927.7.3160 for example, are shown without the common

961 'Tb927.' component.

962

963 Figure 2. Validation and identification of $>1000$ candidates linked to cell cycle defects. (A)

964 The plot on the left shows knockdowns overrepresented in the $>4 \mathrm{C}$ experiment in red; those

965 with $>1.5$-fold the sum of reads in the $G_{1}$, $S$ phase and $G_{2} M$ samples combined. The read-

966 mapping profile and read-counts for $\alpha / \beta$-tubulin are shown to the right. (B) The plot on the

967 left shows knockdowns overrepresented in the sub-2C experiment in orange; those with

$968>1.5$-fold the sum of reads in the $G_{1}, S$ phase and $G_{2} M$ samples combined. The read-

969 mapping profile and read-counts for DOT1A are shown to the right. (C) The plots on the left

970 shows knockdowns overrepresented in the $G_{1}, S$ phase and $G_{2} M$ experiments in purple,

971 green and blue, respectively; those that were $>25 \%$ overrepresented in each category.

972 Read-mapping profiles and relative read-counts for example hits are shown to the right.

973 PCNA, proliferating cell nuclear antigen; PPL2, PrimPol-like 2. (D) The Venn diagram shows

974 the distribution of knockdowns overrepresented in each arm of the screen.

975

976 Figure 3. Cytokinesis defects associated with endoreduplication. (A) The bar-graph shows

977 enriched Gene Ontology terms in the $>4 C$ overrepresented dataset. (B) The violin plot

978 shows relative $>4 \mathrm{C}$ read-counts for cohorts of genes and reflects data distribution. Open

979 circles indicate median values and the vertical bars indicate 95\% confidence intervals.

980 Significantly overrepresented cohorts are indicated in red. (C) The plots show

981 overrepresentation of T-complex, dynein and intraflagellar transport (IFT) factors in red in the

$982>4$ C experiment. (D) The heatmaps show relative representation in all five sorted pools for

983 the above and additional cohorts of knockdowns; blue, most overrepresented. (E) Example

984 read-mapping profiles for hits overrepresented in the $>4 \mathrm{C}$ pool. (F) Example read-mapping

985 profiles for ciliopathy-associated hits overrepresented in the $>4 \mathrm{C}$ pool. CMF, Component of

986 Motile Flagella; CFAP, Cilia and Flagella Associated Protein. 
Figure 4. Defects producing sub-diploid cells. Read-mapping profiles and read-counts for example hits overrepresented in the $<2 \mathrm{C}$ experiment. HPC, Hypothetical Protein,

990 Conserved.

991

992 Figure 5. A profile of $\mathrm{G}_{1}, \mathrm{~S}$ phase and $\mathrm{G}_{2} /$ mitosis defects. (A) The RadViz plot shows 993 knockdowns that registered $>25 \%$ overrepresented read-counts in the $G_{1}$, S phase or $G_{2} M$ categories. (B) The bar-graphs shows enriched Gene Ontology terms in the $G_{1}, S$ phase or $G_{2} M$ overrepresented datasets. (C) The violin plots show relative $G_{1}$, S phase or $G_{2} M$ readcounts for cohorts of genes and reflects data distribution. Open circles indicate median values and the vertical bars indicate 95\% confidence intervals. Overrepresented cohorts are indicated in purple, green and blue, respectively.

(D) The heatmaps show relative representation in all five sorted pools for the above and additional cohorts of knockdowns; blue, most overrepresented.

Figure 6. Protein complexes and pathways associated with $\mathrm{G}_{1}, \mathrm{~S}$ phase and $\mathrm{G}_{2} /$ mitosis defects. (A) The RadViz plot shows glycolytic enzyme knockdowns. Those that registered $>25 \%$ overrepresented read-counts in the $\mathrm{G}_{1}$ category are indicated, purple. Black datapoints indicate other genes from each cohort. Grey data-points indicate all other genes. The read-mapping profiles and relative read-counts in the lower panel show example hits. (B) As in a but for DNA replication initiation factor knockdowns that registered $>25 \%$ overrepresented read-counts in the S phase category, indicated in green. (C) As in a but for proteasome component knockdowns that registered $>25 \%$ overrepresented read-counts in the $\mathrm{G}_{2} \mathrm{M}$ category, indicated in blue. (D) As in a but for kinetochore component knockdowns that registered $>25 \%$ overrepresented read-counts in the $S$ phase or $\mathrm{G}_{2} \mathrm{M}$ categories, indicated in green or blue, respectively.

1014 Figure 7. RBPs kinases and hypothetical proteins associated with gap and S phase defects.

1015 (A) The RadViz plot shows mRNA binding protein knockdowns (RBPs). Those that

1016 registered $>25 \%$ overrepresented read-counts in the $G_{1}$, $S$ phase or $G_{2} M$ categories are

1017 indicated, in purple, green and blue, respectively. The read-mapping profiles and relative

1018 read-counts in the lower panels show example hits. (B) As in a but for protein kinase

1019 knockdowns. (C) As in a but for hypothetical (conserved) protein knockdowns. 


\section{Supplementary Figure Legends}

1022 Figure 1-figure supplement 1. Induced library sorting and RNAi target amplification. (A)

1023 BD Influx ${ }^{\mathrm{TM}}$ cell sorter BD FACSort ${ }^{\mathrm{TM}}$ software workspace data for the sorting session

1024 showing the gates and population frequencies. (B) Flow cytometry quality control data for

1025 the sorted samples. Three types of graphs are shown: SSC-Area x FSC-Area (cell

1026 morphology); PI-Area $\times$ PI-Width (cells stained with PI; the gate excludes cell aggregates);

1027 Modal $\times$ PI-Area (cells within the gate set in the PI-Area $\times$ PI-Width plot). Top row -

1028 unsorted; second row $-G_{1}$; third row $-S$; fourth row $-G_{2} M$; bottom row - overlay of the

1029 sorted and unsorted samples. 2C and 4C refer to unduplicated (diploid genome) and

1030 duplicated DNA content, respectively. (C) PCR amplification of each sorted sample with Lib3

1031 primers, prior to sequencing.

1032

1033 Figure 1-figure supplement 2. RIT-seq data comparing unsorted libraries. (A) The plot

1034 shows read-counts for 7,204 genes and for uninduced and $24 \mathrm{~h}$ induced samples. Reads for only $0.6 \%$ of genes dropped by $>3$-fold following $24 \mathrm{~h}$ of knockdown. (B) The violin plots show relative read-counts for cohorts of genes and reflect data distribution. Open circles indicate median values and the vertical bars indicate 95\% confidence intervals. Read-counts remain relatively high after $24 \mathrm{~h}$ knockdown in the current study, when compared to readcounts after $72 \mathrm{~h}$ knockdown in a prior RIT-seq study.

Figure 2-figure supplement 1. RIT-seq data mapped to T. brucei chromosomes; for 7,204 genes and five experiments $=36,020$ data-points. Light and dark green indicate polycistronic transcription in the forward and reverse directions, respectively, on each chromosome. The heat maps (dark-blue to yellow) indicate rank enrichment for knockdowns in each cell cycle phase; most enriched are dark-blue. The coloured data-points below indicate those 'hits' enriched in each cell cycle phase and used for much of the analysis reported here; 1,158 or $16.1 \%$ of genes. See the text for more details.

Figure 2-figure supplement 2. Online RIT-seq data visualization (https://tryp-

1050 cycle.onrender.com). In the radial visualization, experimental-points are hours on the clock-

1051 face (i.e. related to the angle of the polar coordinate system). The orthogonal axis (i.e. the distance) relates to the relative read-counts across the five experiments. The table on the right shows: Geneid, gene identification number; relative abundance of reads in each sorted sample; desc, gene description; class, the experiment where the gene shows maximum abundance; selected, a binary tag where ' 1 ' indicates genes in the radial visualization. Gene 
coverage images are displayed when hovering over dots on the radial visualization or over table rows.

Data transformation to aid visualization: Normalised TPM values from

Supplementary file 1 were used, except the $<2 \mathrm{C}$ and $>4 \mathrm{C}$ values were divided by the sum of TPM values from all five sorted samples. we elevated the values to the power of 2.1 to maximize differences. We then normalized the values raw wise for each gene, by dividing values by the maximum. The transformed data was then fed to the radial visualization algorithm implemented in D3.js (https://github.com/d3/d3); code and web page were adapted from the repository at https://github.com/WYanChao/RadViz.

Figure 3-figure supplement 1. Cytokinesis defects associated with endoreduplication. One hundred example RIT-seq cell cycle profiles are shown for hits overrepresented in the $>4 \mathrm{C}$ pool. Page 1 shows the heatmaps indicating relative representation in all five sorted pools; blue, most overrepresented. Subsequent pages show read-mapping profiles for each gene; see Figure 1B for further details.

Figure 7-figure supplement 1. Knockdowns associated with gap and S phase defects. Seventy-eight example RIT-seq cell cycle profiles are shown for hits overrepresented in the $G_{1}$, S phase and $G_{2} M$ experiments. Page 1 shows the heatmaps indicating relative representation in all five sorted pools; blue, most overrepresented. Subsequent pages show read-mapping profiles for each gene; see Figure 1B for further details.

Supplementary file 1. RIT-seq digital data. The Excel file reports the total fragment counts for the uninduced (column C) and induced (column D) RNAi libraries, for the five sorted samples (columns E-I), normalised read-counts for the sorted samples (columns J-N) and normalised barcoded read-counts for the G1 (column O), S (column P) and G2M (column Q) samples. The final column $(R)$ indicates Figure numbers for genes shown in the manuscript. TPM, Transcripts Per kilobase Million. Coloured values in column J-Q indicates enrichment in those samples; >1.5-fold the sum of normalised TPM in the G1+S+G2M samples in columns $\mathrm{J}$ and $\mathrm{N},>41.66 \%$ ( $>25 \%$ above the mean) in columns $\mathrm{K}-\mathrm{M}$ and also $>40 \%(>20 \%$ above the mean) in columns O-Q. Genes considered in Figure 2 and used to generate Gene Ontology profiles surpass both the $25 \%$ total reads and $20 \%$ barcoded reads thresholds i.e. coloured in columns O-Q. Data are presented for 7,204 genes, which is $98 \%$ of the nonredundant gene set; all genes register $>99$ total reads across the five sorted samples. 

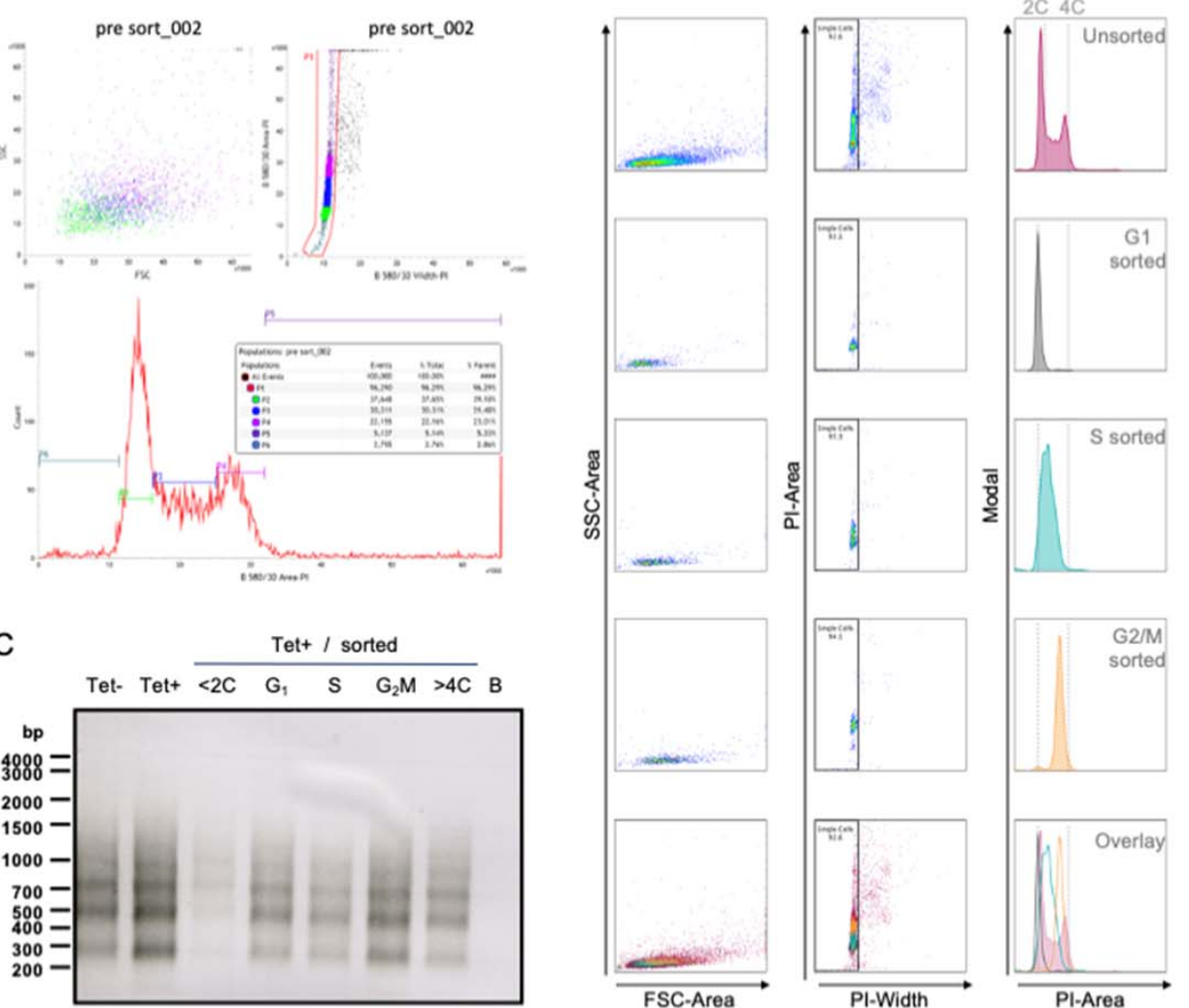

C

Tet+ Telt/ sorted

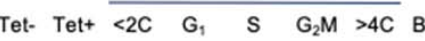
bp
4000
3000
$2000=$
$1500=$
$1000=$
$700=$
$500=$
$400=$
$200=$

Figure 1-figure supplement 1. Induced library sorting and RNAi target amplification. (A) BD Influx TM cell sorter BD FACSort ${ }^{\mathrm{TM}}$ software workspace data for the sorting session showing the gates and population frequencies. (B) Flow cytometry quality control data for the sorted samples. Three types of graphs are shown: SSC-Area $\times$ FSC-Area (cell morphology); PI-Area $\times$ PI-Width (cells stained with PI; the gate excludes cell aggregates); Modal $\times$ PI-Area (cells within the gate set in the PI-Area $\times$ PI-Width plot). Top row - unsorted; second row - $G_{1}$; third row $-S$; fourth row $-G_{2} M$; bottom row - overlay of the sorted and unsorted samples. 2C and $4 \mathrm{C}$ refer to unduplicated (diploid genome) and duplicated DNA content, respectively. (C) PCR amplification of each sorted sample with Lib3 primers, prior to sequencing; B, blank. 
A
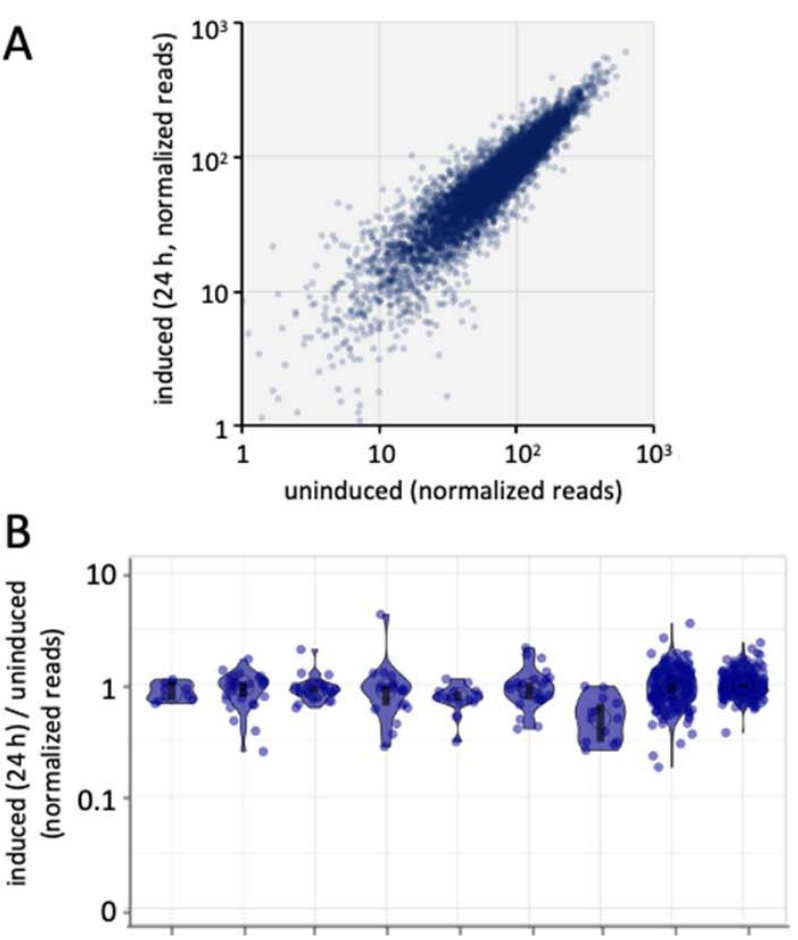

current study

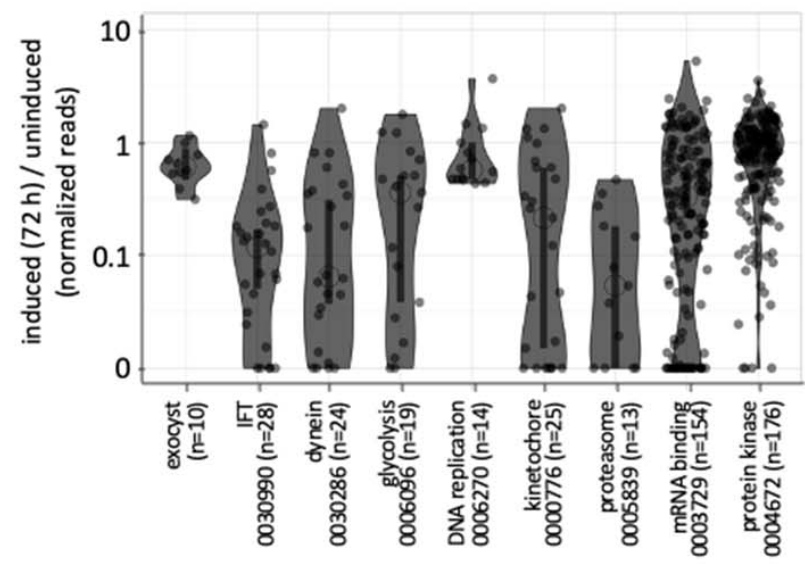

Alsford et al., 2011

Figure 1-figure supplement 2. RIT-seq data comparing unsorted libraries. (A) The plot shows readcounts for 7,204 genes and for uninduced and $24 \mathrm{~h}$ induced samples. Reads for only $0.6 \%$ of genes dropped by $>3$-fold following $24 \mathrm{~h}$ of knockdown. (B) The violin plots show relative read-counts for cohorts of genes and reflect data distribution. Open circles indicate median values and the vertical bars indicate $95 \%$ confidence intervals. Read-counts remain relatively high after $24 \mathrm{~h}$ knockdown in the current study, when compared to read-counts after $72 \mathrm{~h}$ knockdown in a prior RIT-seq study. 


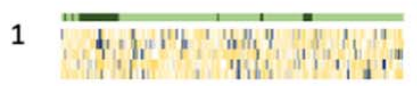

$$
41,1,14,1,1,1,1,
$$

2

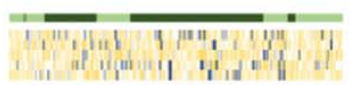

3

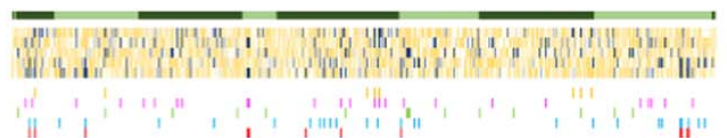

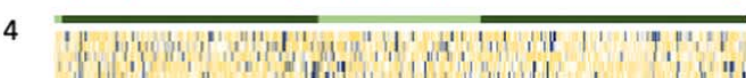

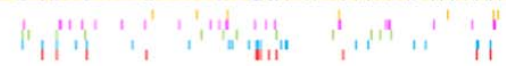

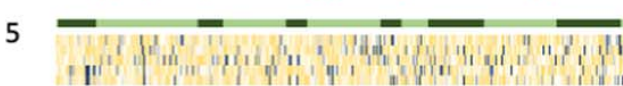

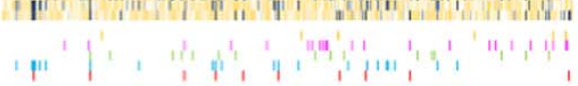

\section{Chromosome}

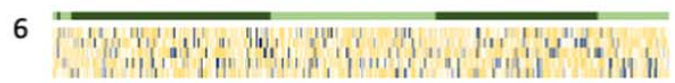

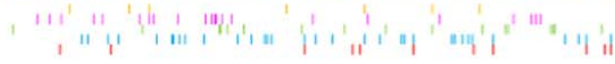

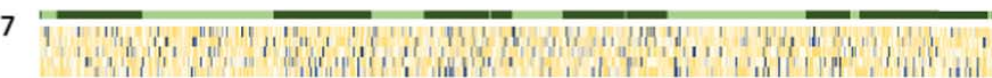
$\therefore<2 \mathrm{C}$

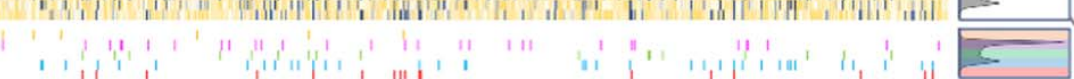
$\mathrm{G}_{1} \quad 1 \quad 7204$ $\mathrm{S} i$ $>4 C$
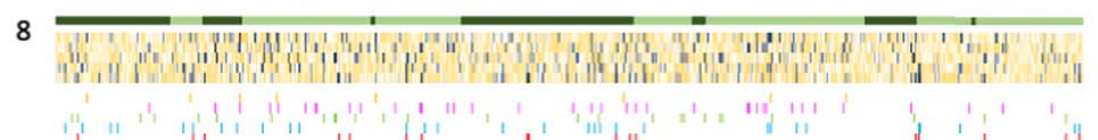

9

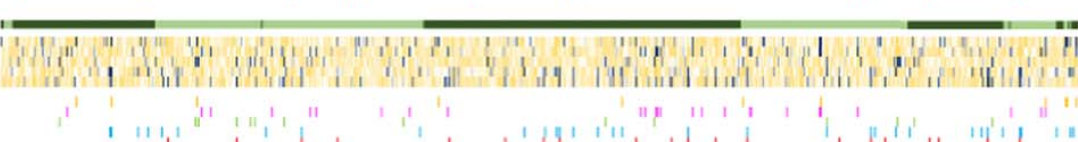

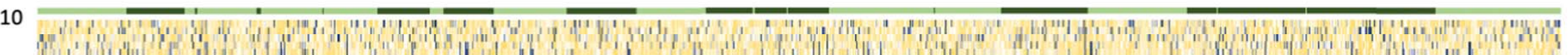

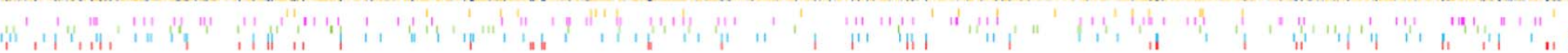

Figure 2-figure supplement 1. RIT-seq data mapped to T. brucei chromosomes; for 7,204 genes and five experiments = 36,020 data-points. Light and dark green indicate polycistronic transcription in the forward and reverse directions, respectively, on each chromosome. The heat maps (dark-blue to yellow) indicate rank enrichment for knockdowns in each cell cycle phase; most enriched are dark-blue. The coloured data-points below indicate those 'hits' enriched in each cell cycle phase and used for much of the analysis reported here; 1,158 or $16.1 \%$ of genes. See the text for more details. 


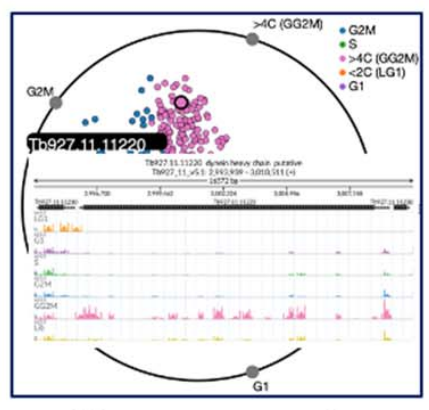

Mouse-over reveals sequence tracks for each gene
The RadViz plot shows 270 'hits' featured in the manuscript

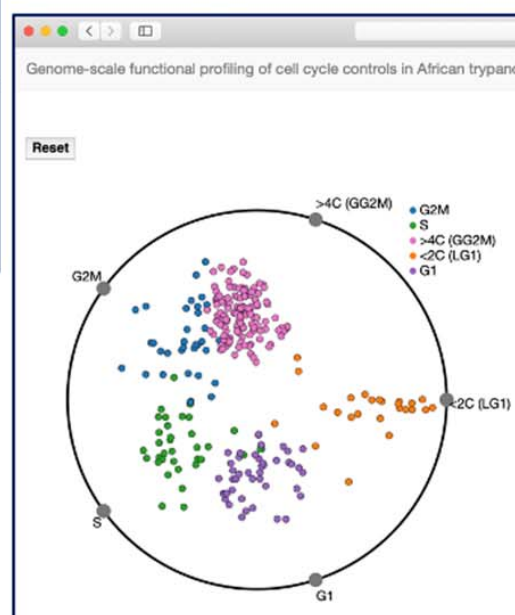

The table lists all 7,204 genes

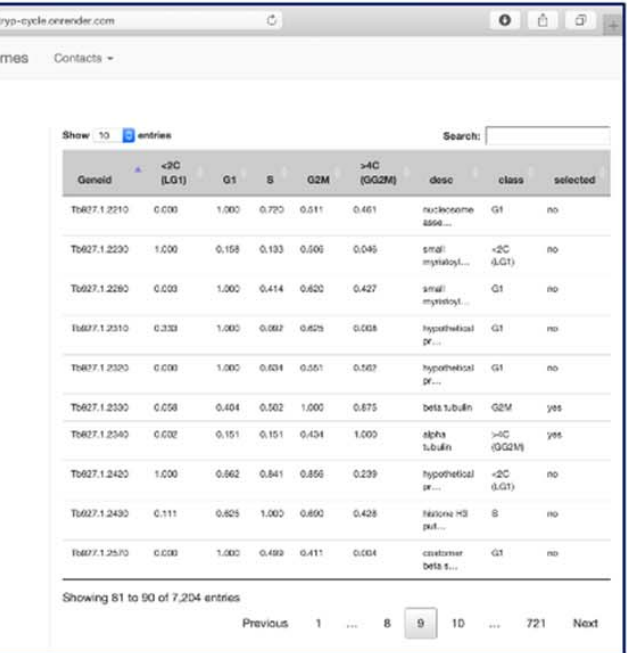

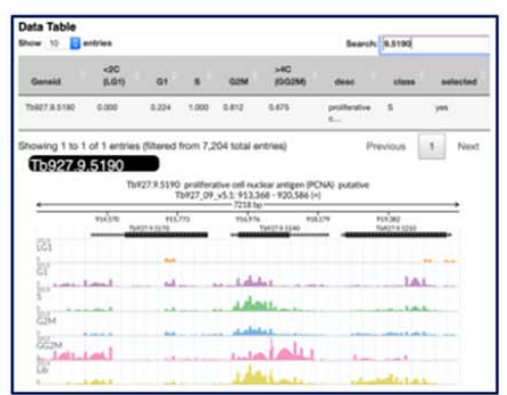

The data can be searched by GeneID or description. Mouse-over also reveals sequence tracks

Figure 2-figure supplement 2. Online RIT-seq data visualization (https://tryp-cycle.onrender.com). In the radial visualization, experimental-points are hours on the clock-face (i.e. related to the angle of the polar coordinate system). The orthogonal axis (i.e. the distance) relates to the relative read-counts across the five experiments. The table on the right shows: Geneid, gene identification number; relative abundance of reads in each sorted sample; desc, gene description; class, the experiment where the gene shows maximum abundance; selected, a binary tag where 'yes' indicates genes in the radial visualization. Gene coverage images are displayed when hovering over dots on the radial visualization or over table rows.

Data transformation to aid visualization: Normalised TPM values from Supplementary file 1 were used, except the $<2 \mathrm{C}$ and $>4 \mathrm{C}$ values were divided by the sum of TPM values from all five sorted samples. we elevated the values to the power of 2.1 to maximize differences. We then normalized the values raw wise for each gene, by dividing values by the maximum. The transformed data was then fed to the radial visualization algorithm implemented in D3.js (https://github.com/d3/d3); code and web page were adapted from the repository at https://github.com/WYanChao/RadViz. 

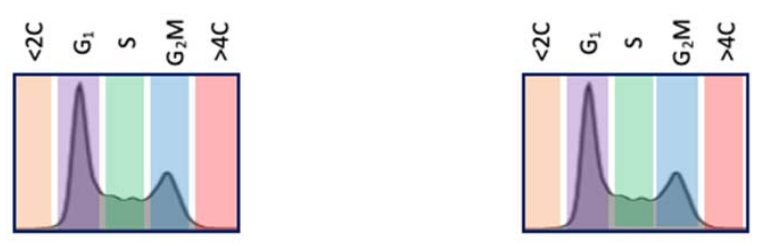

$$
i_{\text {rank order }}^{7204}
$$
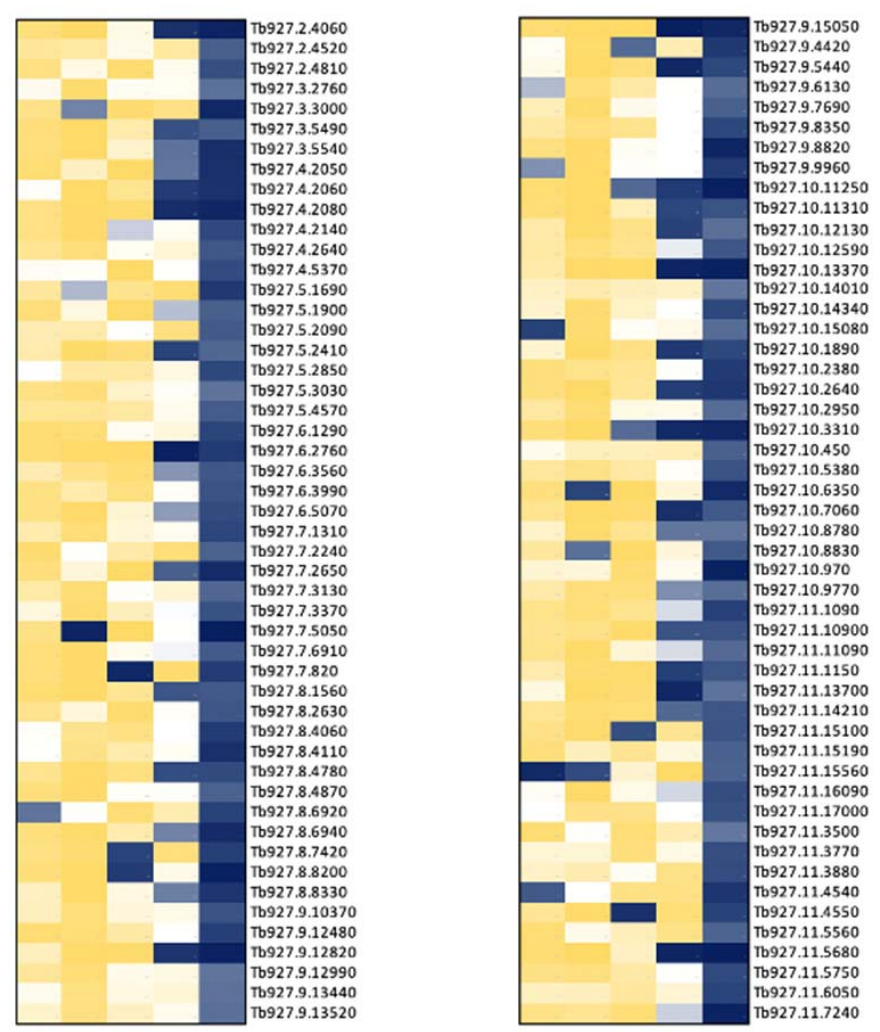

Figure 3-figure supplement 1. Cytokinesis defects associated with endoreduplication. One hundred the heatmaps, indicating relative representation in all five sorted pools; blue most overrepresented. Subsequent pages show read-mapping profiles for each gene; see Figure $1 \mathrm{~B}$ for further details. 

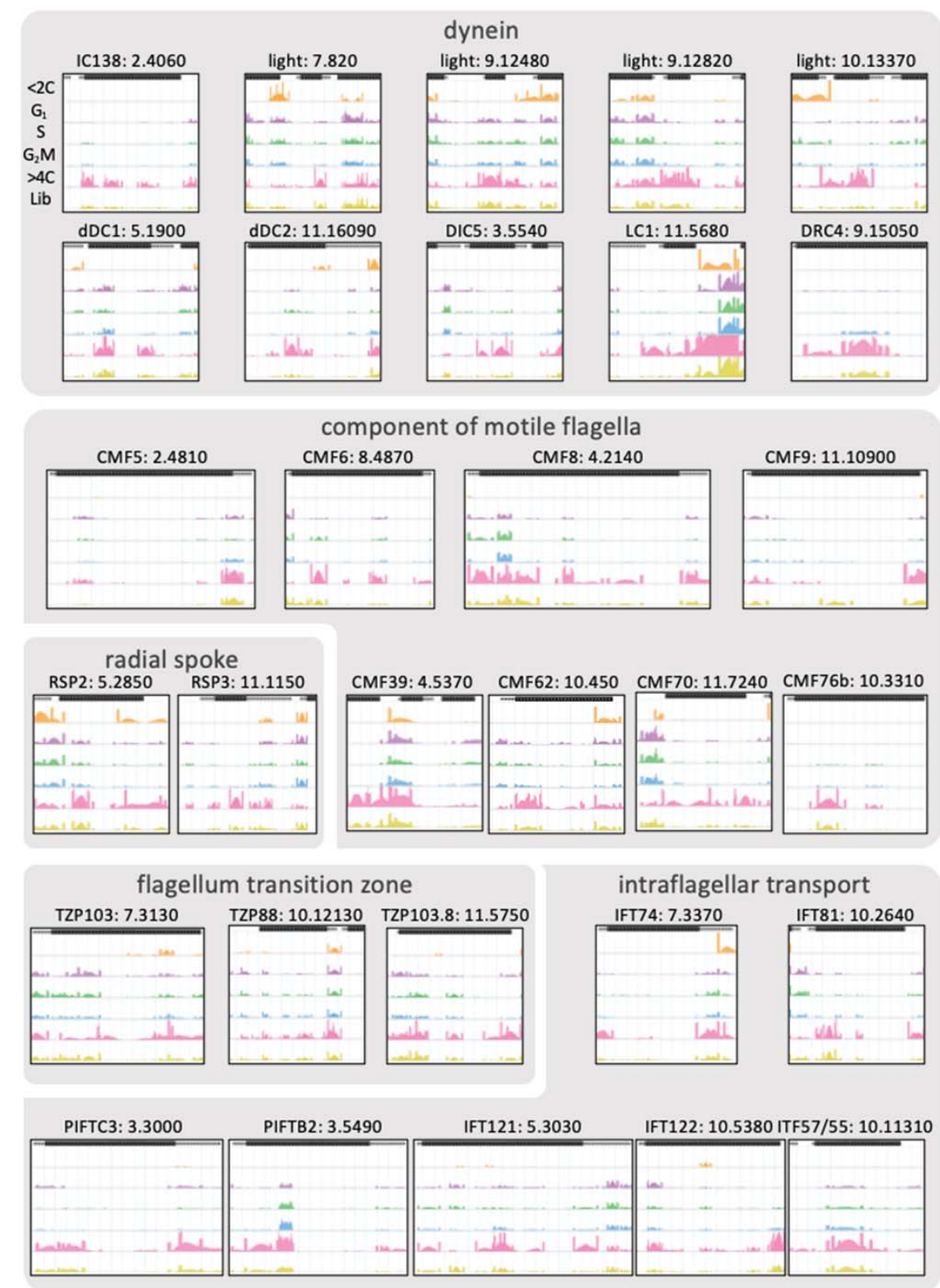

Figure 3 - figure supplement 1 - p2 of 5 
flagellar attachment
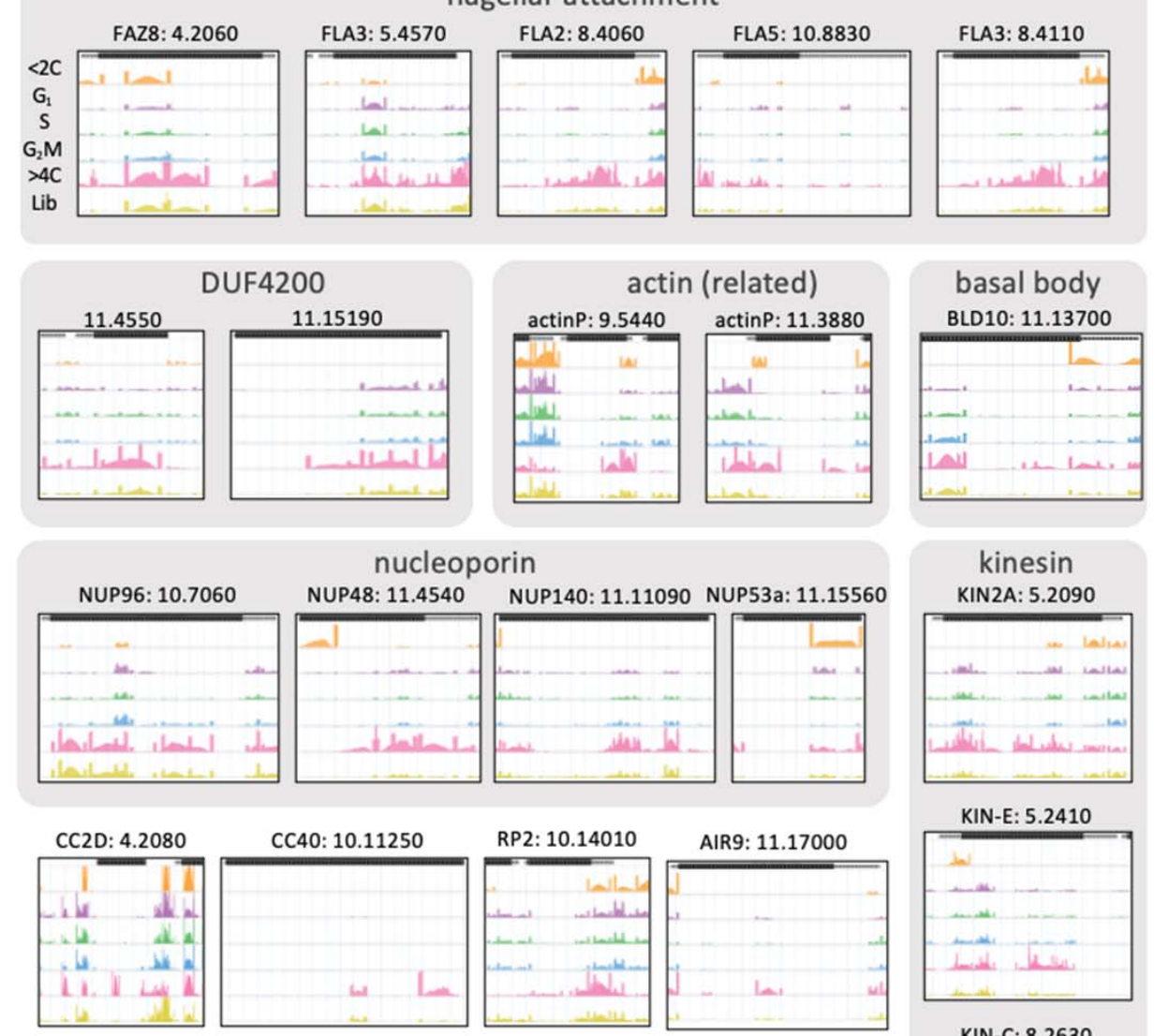

CC40: 10.11250

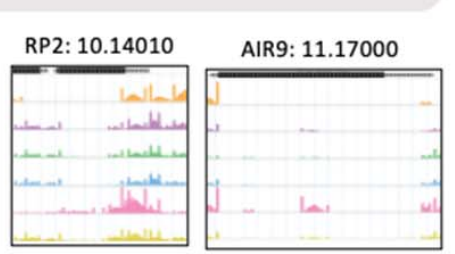

KIN-E: 5.2410

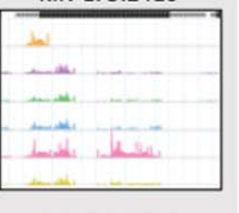

CAP51V: 7.2650

CAP5.5V: 8.8330

FLAM3: 8.4780

calmodulin: 9.6130

KIN-C: 8.2630
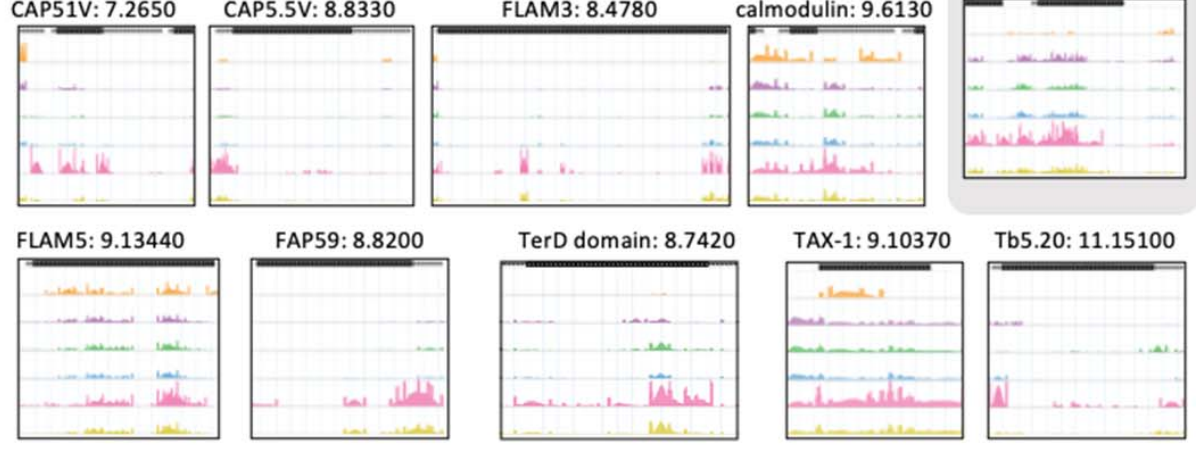

Figure 3 - figure supplement $1-p 3$ of 5 


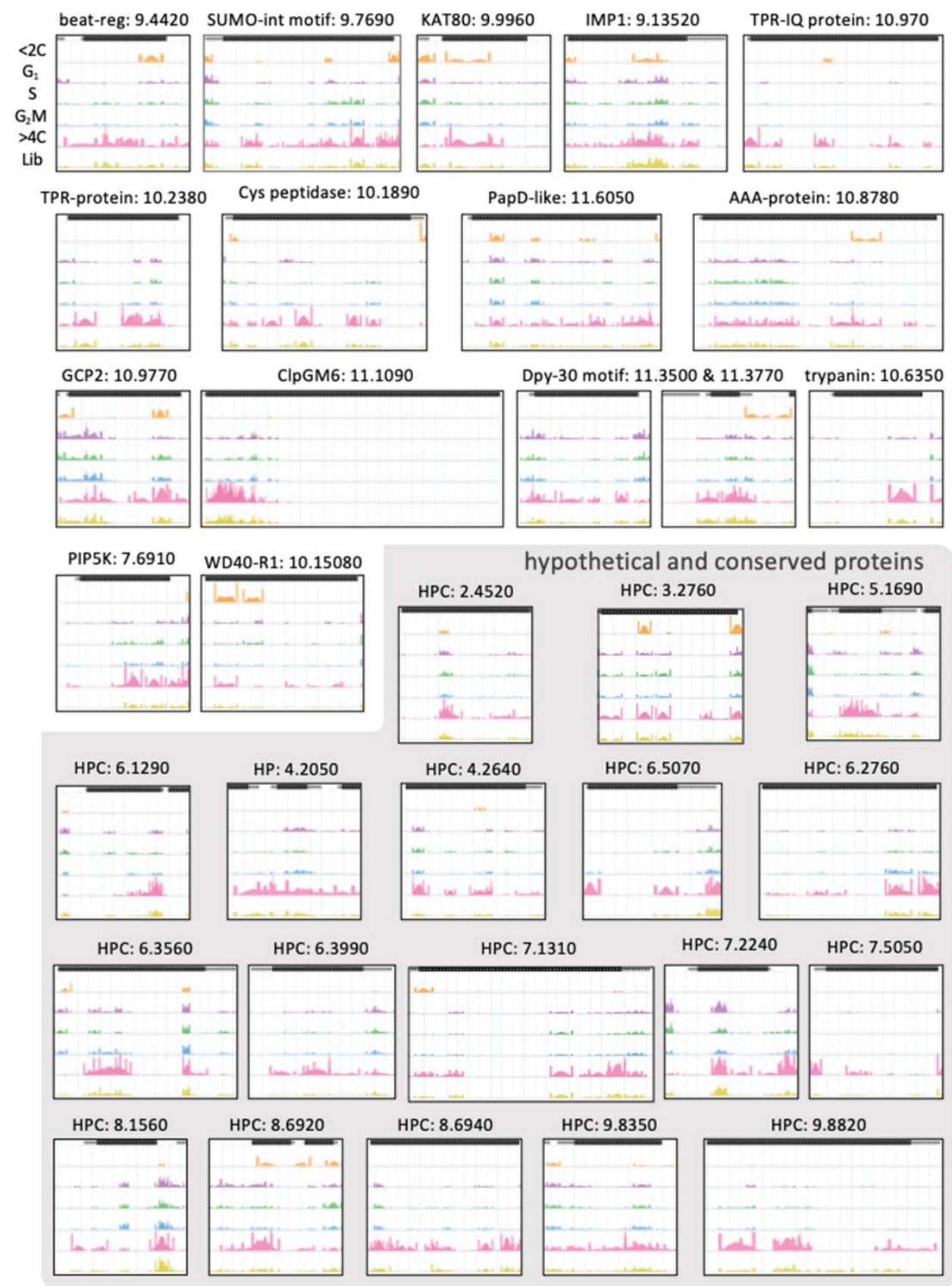

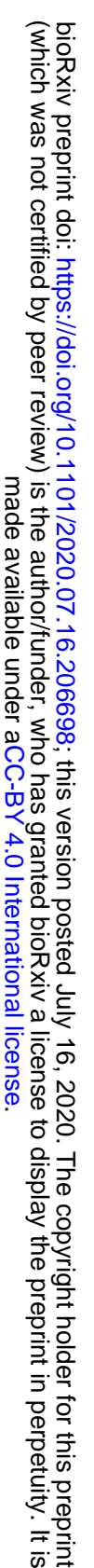

Figure 3 - figure supplement $1-p 4$ of 5 


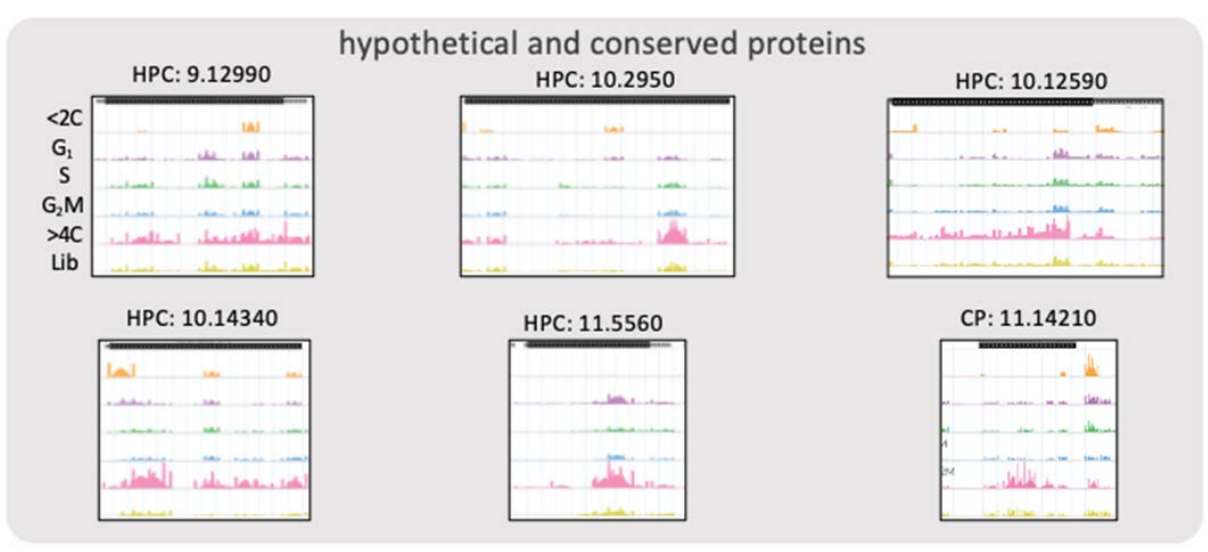

Figure 3 - figure supplement $1-p 5$ of 5 

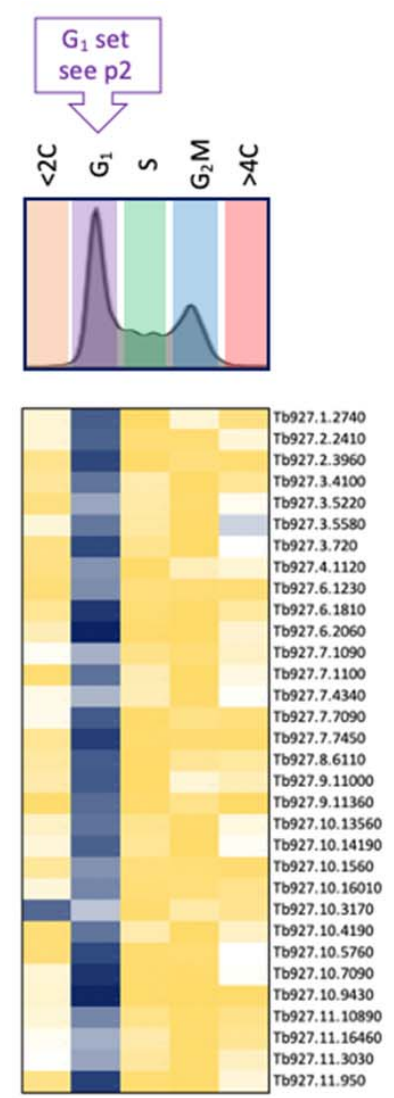

To927.11.3030
ro927.11.950
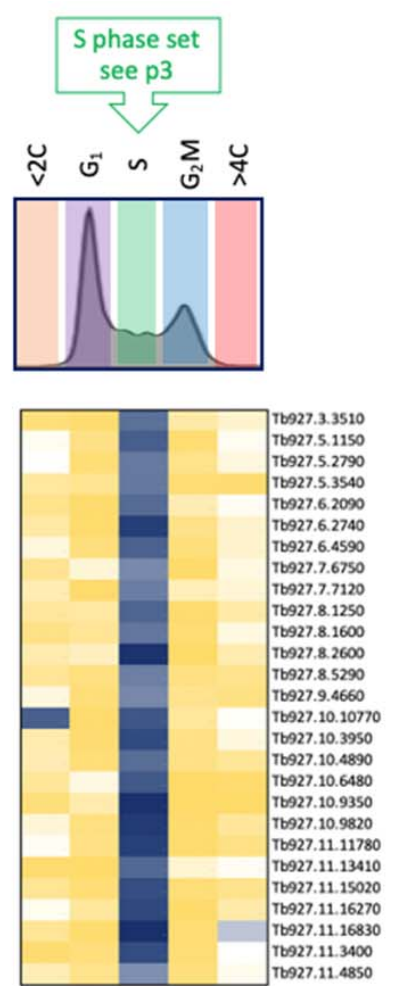
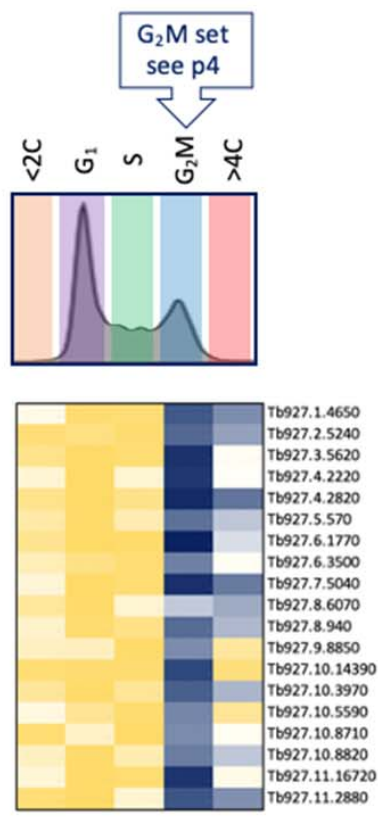

${ }_{\text {rank order }}^{1} 7204$

Figure 7-figure supplement 1. Knockdowns associated with gap and $S$ phase defects. Seventy-eight example RIT-seq cell cycle profiles are shown for hits overrepresented in the $\mathrm{G}_{1}$, $S$ phase and $G_{2} M$ experiments. Page 1 shows the heatmaps indicating relative representation in all five sorted pools; blue, most overrepresented. Subsequent pages show read-mapping profiles for each gene; see Figure 1B for further details. 
Enriched in $\mathrm{G}_{1}$
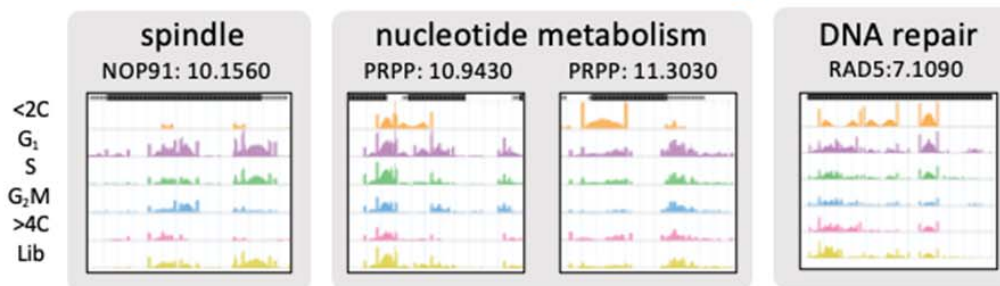

phosphatases

RNA-binding
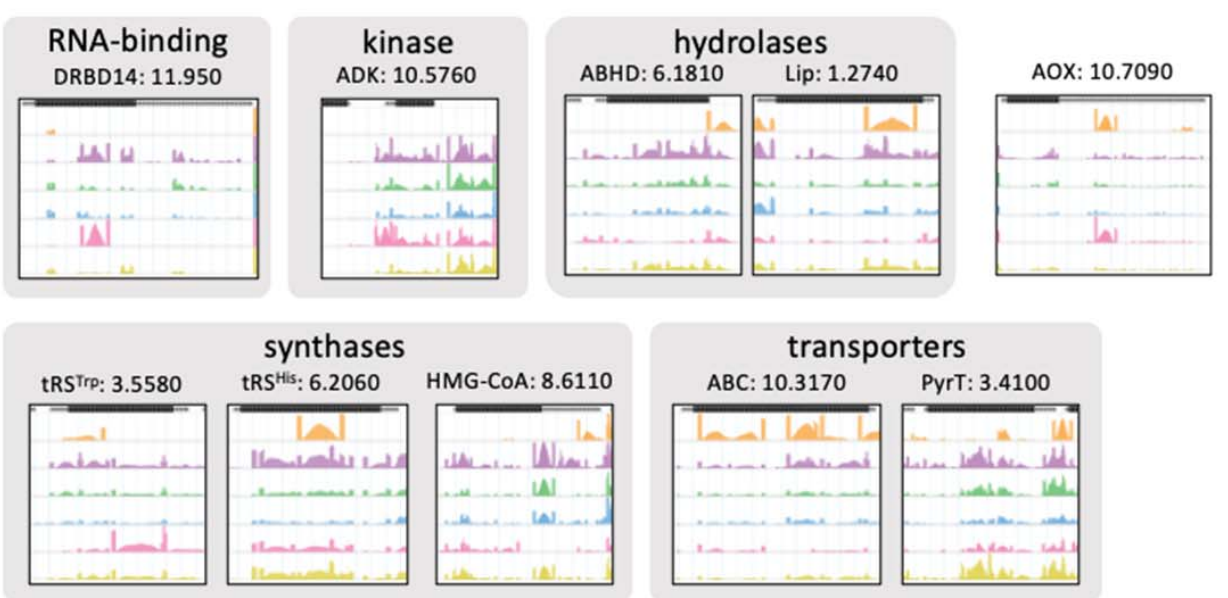

synthases

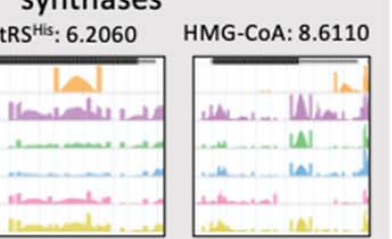

transporters
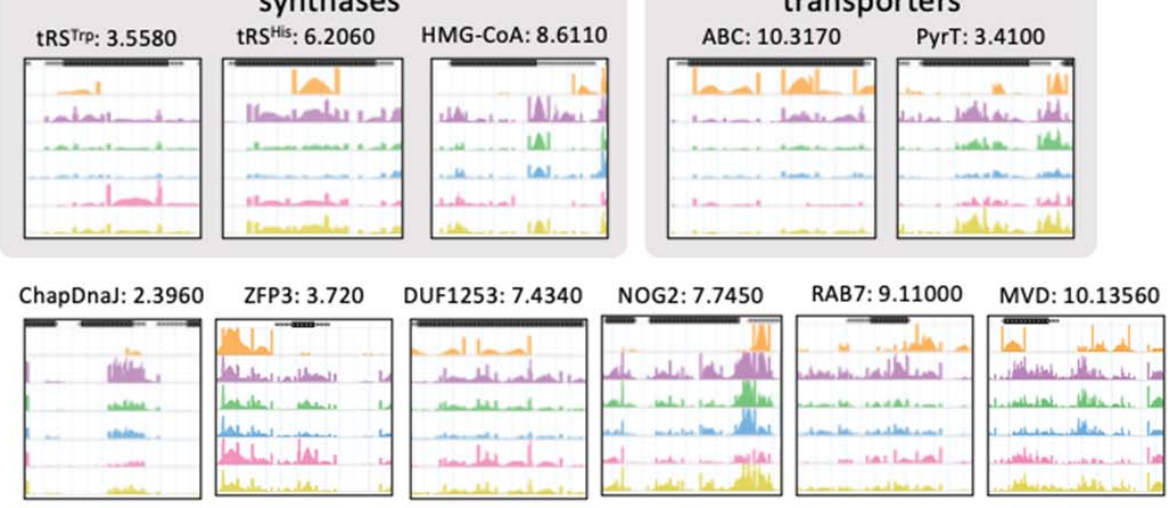

hypothetical and conserved proteins

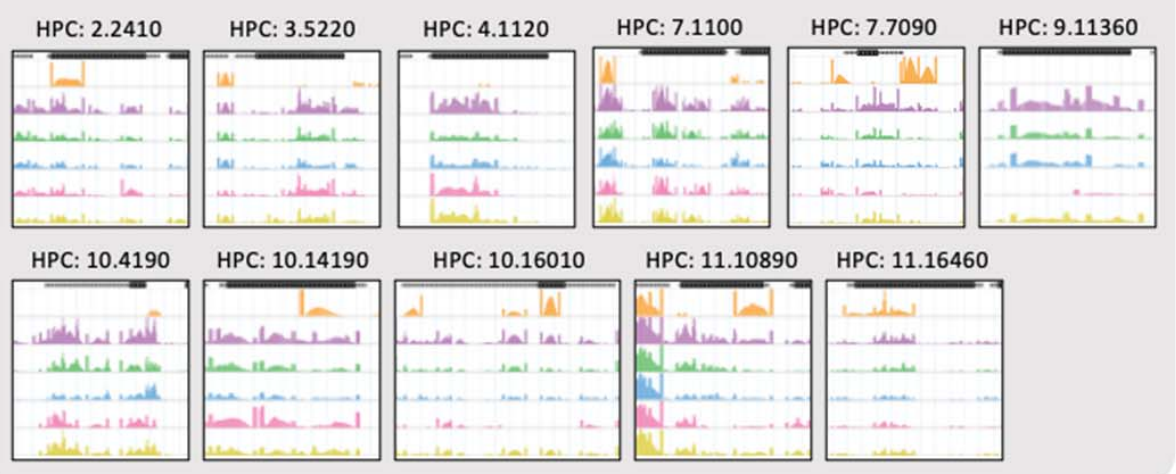

\begin{tabular}{|c|c|c|}
\hline HPC: 10.16010 & HPC: 11.10890 & HPC: 11.16460 \\
\hline $\mathbb{A}$ & 1 & Lethe \\
\hline & Whandina & 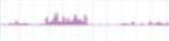 \\
\hline …. & if wiven. & Lench \\
\hline 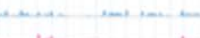 & 7. & - \\
\hline & & \\
\hline
\end{tabular}

Figure 7 - figure supplement 1 - p2 of 4 

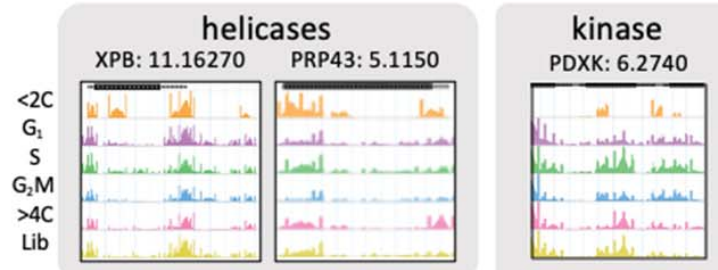

\section{GTPases} RABL5: 11.16830

peptidases

i

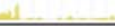

(n)

तit a w

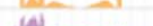

$-\frac{14}{4}$

tRNA synthetases

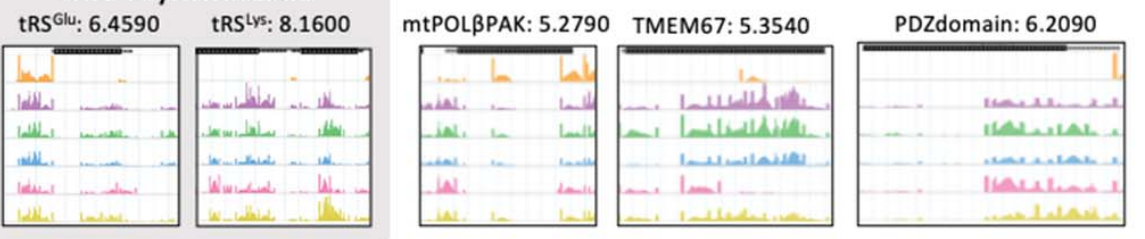

\begin{tabular}{|c|c|c|c|c|c|}
\hline kinesin: 7.7120 & SEL1r: 8 . & NUC141: 8.2600 & WW: 8.5290 & $\mathrm{ZnfC} 2 \mathrm{HC}: 10.4890$ & GCS1: \\
\hline & & & & & الهـا \\
\hline 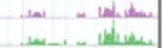 & | & LิA & 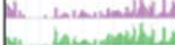 & $-m a t=1$ & . \\
\hline 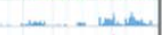 & 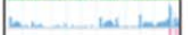 & 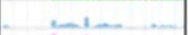 & 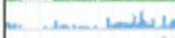 & 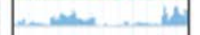 & $\ldots$ \\
\hline 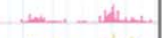 & 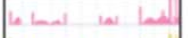 & Lal & al & 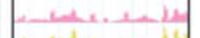 & Lall \\
\hline
\end{tabular}

ACAD: 11.11780 Fe-SOD: 11.15020
HPC: 3.3510
HPC: 10.6480




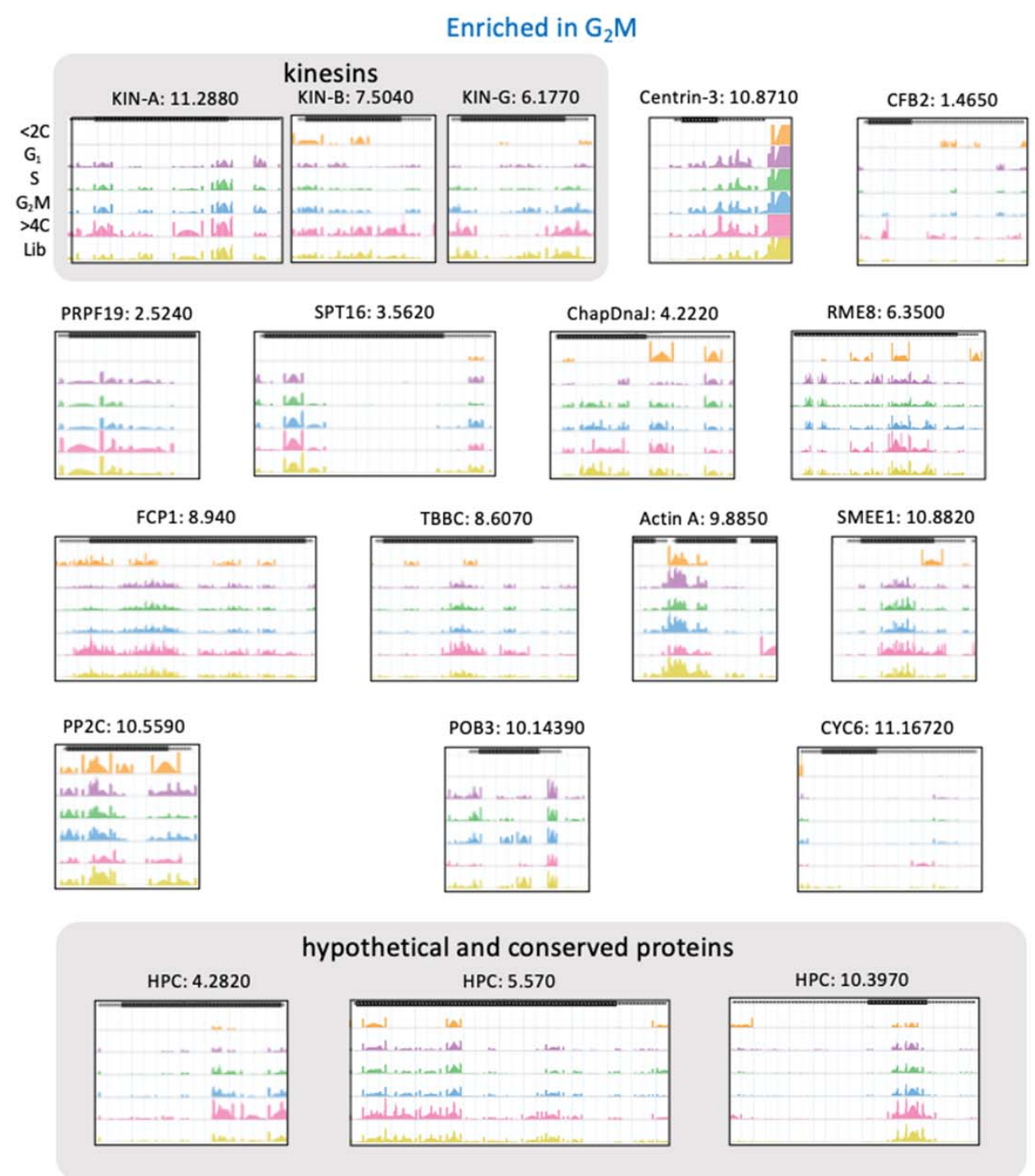

Figure 7-figure supplement 1-p4 of 4 